\title{
Multiorbital electronic correlation effects of Co adatoms on graphene: An ionic Hamiltonian approach
}

\author{
M. S. Tacca $\odot,{ }^{1,2}$ T. Jacob, ${ }^{2}$ and E. C. Goldberg ${ }^{1}$ \\ ${ }^{1}$ Instituto de Física del Litoral (CONICET-UNL), Güemes 3450, S3000GLN Santa Fe, Argentina \\ ${ }^{2}$ Institute of Electrochemistry, Ulm University, Albert-Einstein-Allee 47, D-89081 Ulm, Germany
}

(Received 21 January 2020; revised manuscript received 28 February 2020; accepted 2 March 2020; published 18 March 2020)

\begin{abstract}
In the present work, we propose an ionic Hamiltonian for describing the interaction of graphene with an adsorbed Co atom. In this approach, the electronic correlation effects, related to the many $d$ orbitals involved in the interaction, are taken into account by selecting appropriate electronic configurations of the adsorbed atom. The Hamiltonian parameters are calculated considering the localized and extended features of the atom-surface interacting system. The physical quantities of interest are calculated by using a Green functions formalism, solved by means of the equations of motion method closed up to a second order in the atom-band coupling term. The charge and spin fluctuations in the adsorbed Co atom are inferred from density functional theory calculations and assuming that the lower energy configurations obey Hund's rules. The calculated spectral densities and the occurrence probabilities of the different atomic configurations are analyzed as a function of the Co energy level positions and the surface temperature. In addition, the conductance spectra are calculated by using the Keldysh formalism and compared with existing measurements. We analyze the behavior, under variable bias and gate potentials, of resonancelike features in the conductance spectra which can be related to transitions between atomic configurations of low occurrence probability.
\end{abstract}

DOI: 10.1103/PhysRevB.101.125419

\section{INTRODUCTION}

Considerable effort has been put into the development of graphene-based devices for multiple applications [1]. Graphene has the property of allowing spin transport with high efficiency and long relaxation distances [2]. Nevertheless, the diamagnetic nature of graphene requires some method to modify its electronic structure, in order to use it in technological applications. The various methods include the adsorption of magnetic impurities [3], the doping and generation of defects [4] and the growing on different substrates [5], among others [1]. It is also of interest to tune the induced changes in the electronic structure, for example by gating or doping. Besides the potential of $\mathrm{Co}$ and other transition metals adsorbed in graphene for spintronic applications $[1,6,7]$, the interest in these systems comes from the possibility of observing exotic Kondo physics [8-10].

The Co on graphene system has been the focus of many theoretical $[8,11-17]$ and experimental $[6,18]$ works. The combination of the multiorbital features proper of the Co $d$ valence shell and the particular characteristics of graphene electronic structure makes the system both interesting and challenging. The conductance spectra obtained by using scanning tunnelling microscopy (STM) [18] reveal many unidentified structures which have been object of different theoretical interpretations $[8,13,14,18]$.

The theoretical description of this system is still under discussion. At the density functional theory (DFT) level, the most stable position is found to be at the hollow site, with an adsorption height of $\approx 1.5 \AA$ with either generalized gradient (GGA) or local density (LDA) approximations $[3,8,11,12,14,19-21]$. Nevertheless, when the GGA $+U$ method [22] is used, the results are quite sensitive to the value of $U$. For example, for $U=4 \mathrm{eV}$, the most stable position is on top at $\approx 1.8 \AA[3,11,12,14]$. More possibilities are found by using different functionals $[8,13]$. The electronic configurations found in the literature are $3 d^{9} 4 s^{0}$ with total spin $S=1 / 2$ [3,12], $3 d^{8} 4 s^{1}$ with $S=3 / 2[3,11,12]$ and an intermediate occupation of 7.5 in the $d$ shell due to a dynamical hybridization with graphene [8]. In addition, a cluster calculation based on a wave-function approach [15] predicted the configurations $3 d^{9} 4 s^{0}$ and $3 d^{7} 4 s^{2}$ at Co-graphene distances of 1.6 and $3.1 \AA$, respectively. A stochastic approach $[16,17]$ also suggested the configurations $3 d^{9} 4 s^{0}$ and $3 d^{7} 4 s^{2}$ at approximately the same distances, with an intermediate $3 d^{8} 4 s^{1}$ configuration at $\approx 1.8 \AA$.

The system was also analyzed by using the Anderson impurity model [23]. In equilibrium situations, the Anderson model can be solved by using different approaches, such as quantum Monte Carlo (QMC), numerical renormalization group (NRG) [24,25], or the exact diagonalization (ED) approach [26]. In out-of-equilibrium situations, adequate approximations are necessary to describe the system properties. The most commonly used are probably the noncrossing and one-crossing approximations (NCA and OCA) [27-29], although the equations of motion (EOM) approach [30,31], used in the present work, provides a useful alternative [32-35]. In this context, the Co on graphene system was solved at several temperatures by using the GGA+OCA method [8] and recently by using both QMC and ED [11]. In addition, the 
system was analyzed by using a tight-binding approximation and a renormalization approach [12].

The goal of this paper is the description of the Co on graphene system in the framework of the Anderson Hamiltonian in its ionic form $[9,36]$. Our results suggest a mixed valence regime involving $3 d^{8} 4 s^{1}$ and $3 d^{7} 4 s^{1}$ electronic configurations, which is consistent with the available studies. An advantage of the ionic Hamiltonian approach proposed in the present work is that it provides the contribution of each configuration to the orbital occupations and spectral densities In Sec. II, we present the DFT calculations performed to obtain the equilibrium positions of the adsorbate and the orbital occupations. Based on these results and taking into account Hund's rules, in Sec. III A, we select a suitable space of configurations to project the Anderson Hamiltonian. In Sec. III B, we introduce the ionic Hamiltonian projected into the $\mathrm{Co}$ electronic configurations that have a significant occurrence probability according to the DFT calculations.

Appropriate Keldysh-Green functions [37] are introduced for calculating the physical quantities of interest in equilibrium and out of equilibrium. These Green functions are calculated by employing the EOM method closed up to second order in the coupling between the atom and the surface [32]. This approach has already been used to describe several many-body systems, with a good qualitative description of the Kondo physics and the inelastic excitations in out-ofequilibrium processes [32,38-40]. The details of the resolution method are presented in Sec. III C, while symmetry considerations are discussed in Sec. III D.

The bond-pair model [41] is used to determine the Hamiltonian parameters: the one electron energy levels involved in the charge transfer process and the atom-surface coupling terms leading to the hybridization functions. The calculation of these parameters is presented and analyzed in Sec. IV. The main advantages of this proposal are the absence of supercell effects, the possibility of using a primitive cell for the surface calculation and a well-defined double counting correction for the energy level calculation. The orbital occupations and spectral densities at several temperatures and energy level positions and the effect of restricting the selected configuration space are discussed in Sec. IV. We find a good agreement with previous results, presenting new insights provided by our model. We show that the selected configuration space suppresses the fluctuation of the most occupied orbital for certain energy level positions. Spectral density structures that either appear or are enhanced when the temperature increases are straightforwardly identified with charge fluctuations that become accessible. These structures are present in previous works, but their origin was not explored. In Sec. VI, we employ the Keldysh formalism to calculate the differential conductance spectra for different values of gate potentials and energy levels. We propose that some resonancelike features observed in the measurement are related to fluctuations between configurations with low probability of occurrence. The conclusions of this work are presented in Sec. VII.

\section{DFT CALCULATION: EQUILIBRIUM GEOMETRY AND ORBITAL OCCUPATION}

We begin our study with standard DFT calculations on the system, as is usual in different approaches aimed at
TABLE I. DFT Orbital occupations per spin for the non-spinpolarized (nsp) and spin-polarized (sp) DFT calculations, for the majority $(\uparrow)$ and minority $(\downarrow)$ spin. The total occupation in the $d$, $s$, and $p$ shells are presented.

\begin{tabular}{lcccccccc}
\hline \hline Case & $A 1$ & $E 1$ & $E 2$ & $d$ shell & $s$ & $p_{x / y}$ & $p_{z}$ & $s+p$ shell \\
\hline nsp & 0.98 & 0.63 & 0.81 & 3.86 & 0.13 & 0.13 & 0.08 & 0.47 \\
sp $\uparrow$ & 0.98 & 0.94 & 0.88 & 4.62 & 0.13 & 0.15 & 0.08 & 0.51 \\
sp $\downarrow$ & 0.98 & 0.37 & 0.79 & 3.30 & 0.13 & 0.10 & 0.08 & 0.41 \\
\hline \hline
\end{tabular}

improving the treatment of electronic correlation effects $[8,11,12,29,42,43]$. For this purpose, we used the SEQQUEST code $[44,45]$, based on the linear combination of atomic orbital (LCAO) method, to obtain the equilibrium geometry of the system, the local density of states (LDOS) and the occupation of the Co atom. The DFT results allow us to infer the electronic configurations of the adsorbed Co atom that will define the ionic Hamiltonian.

We used a $6 \times 6$ supercell, with a vacuum separation of $15 \AA$ to ensure the decoupling in the direction normal to the surface. For exchange and correlation the PBE $[46,47]$ functional was used, while the geometry was optimized until the forces were smaller than $0.01 \mathrm{eV} / \AA$. After relaxation, the most favorable configuration was the Co adatom in the hollow position, with an equilibrium distance to the graphene sheet of $1.52 \AA$, in agreement with previous results $[3,8,11,12,14,19$ $21]$. We computed the density of states and orbital occupations using a $28 \times 28 \times 1 \mathbf{k}$ grid and performing DFT studies both with and without spin polarization.

The C6 symmetry of the system induces a splitting of the $5 d$ orbitals of the Co atom into the groups $A 1\left(d_{z^{2}} \equiv d_{1}\right)$, $E 1\left(d_{x z} \equiv d_{2}\right.$ and $\left.d_{y z} \equiv d_{3}\right)$ and $E 2\left(d_{x y} \equiv d_{4}, d_{x^{2}-y^{2}} \equiv d_{5}\right)$. In Table I, we present the orbital occupations in the $d$ shell for each symmetry group and in the $s$ and $p$ orbitals, for the nonspin-polarized (nsp) and spin-polarized (sp) DFT calculations.

The $s$ and $p$ orbital occupations are low, $\approx 0.1$ electrons per spin, and their contribution to the LDOS of Co atom around the Fermi energy is negligible. Therefore it is not expected that these orbitals contribute to charge or spin fluctuations.

The DFT calculations predict a total occupation in the $d$ shell equal to 7.72, suggesting a charge fluctuation between 7 and 8 electrons. The total occupations per group ( $A 1: 1.96$, $E 1: 2.52, E 2: 3.24)$ suggest that $A 1$ is completely occupied, $E 1$ fluctuates between 2 and 3 electrons, and $E 2$ between 3 and 4 electrons. The results for the spin-polarized calculation formally lead to the same conclusions.

\section{HAMILTONIAN AND RESOLUTION METHOD}

\section{A. Space of atomic configurations}

Based on the DFT results presented in Sec. II, we propose a charge fluctuation between 7 and 8 electrons in the $d$ shell. In the hole picture, which is used in this work, the fluctuations are between 3 and 2 holes. According to Hund's rules, the total spin values $S=3 / 2$ and $S=1$ define the lower energy configurations involved in this fluctuation. These $|S, M\rangle$ states, being $M$ the spin projection, are written in terms of the atomic configurations $\left|d_{1}(A 1), d_{2}(E 1) d_{3}(E 1), d_{4}(E 2) d_{5}(E 2)\right\rangle$. Ten 
electronic configurations result for $S=3 / 2$ and $M=3 / 2$ from the different possibilities of filling the five $d$ orbitals with three holes with spin $\uparrow$. In a similar way, ten configurations with $S=1$ and $M=1$ are built by filling the five $d$ orbitals with two holes with spin $\uparrow$. The other configurations are obtained by applying the ladder operator $\hat{S}_{-}$to those with maximum value of $M$. In absence of an applied magnetic field and neglecting the spin-orbit interaction, the states $|S, M\rangle$ are degenerated in $M$.

We label the $|S, M\rangle$ states with indices $p=d_{a} d_{b} d_{c}$ (for $S=3 / 2$ ) and $q=d_{a} d_{b}$ (for $S=1$ ), to identify the orbitals occupied with holes. For example, the state $\left|\frac{3}{2}, \frac{3}{2}\right\rangle_{d_{1} d_{2} d_{3}}=$ $|\uparrow, \uparrow \uparrow, 00\rangle$ fluctuates to $|1,1\rangle_{d_{2} d_{3}}=|0, \uparrow \uparrow, 00\rangle$ when the hole in the $d_{1}$ orbital $(A 1)$ is transferred to the substrate. We use the notation $d(p, q)$ to indicate the active orbital in the transition between the configurations labeled with $p$ and $q$. In the previous example, $d\left(d_{1} d_{2} d_{3}, d_{2} d_{3}\right)=d_{1}$.

\section{B. Ionic Hamiltonian}

The normalization condition of the configuration space restricted to fluctuations between $S=3 / 2$ and $S=1$ is the following:

$$
\sum_{M, q}|1, M\rangle_{q}\left\langle 1,\left.M\right|_{q}+\sum_{M, p} \mid \frac{3}{2}, M\right\rangle_{p}\left\langle\frac{3}{2},\left.M\right|_{p}=\hat{1},\right.
$$

where we used the Hubbard projection operators $|S, M\rangle\langle S, M|$ [48]. We write the Anderson Hamiltonian in the ionic form by projecting it in the selected space of atomic configurations [9]:

$$
\begin{aligned}
\hat{H}= & \sum_{\mathbf{k}, \sigma} \epsilon_{\mathbf{k}} n_{\mathbf{k} \sigma}+\sum_{M, q} E_{1, q}|1, M\rangle_{q}\left\langle 1,\left.M\right|_{q}\right. \\
& +\sum_{M, p} E_{3 / 2, p}\left|\frac{3}{2}, M\right\rangle_{p}\left\langle\frac{3}{2},\left.M\right|_{p}\right. \\
& +\sum_{\mathbf{k}, \sigma, M, p, q}\left(V_{\mathbf{k} M \sigma}^{p q} c_{\mathbf{k} \sigma}^{\dagger}|1, M-\sigma\rangle_{q}\left\langle\frac{3}{2},\left.M\right|_{p}+\text { H.c. }\right) .\right.
\end{aligned}
$$

Here, $n_{\mathbf{k} \sigma}=c_{\mathbf{k} \sigma}^{\dagger} c_{\mathbf{k} \sigma}$ is the number operator, with $c_{\mathbf{k} \sigma}^{\dagger}\left(c_{\mathbf{k} \sigma}\right)$ the operator that creates (annihilates) a hole with spin projection $\sigma= \pm 1 / 2$ in the $\mathbf{k}$-band state. The energy of the $\mathbf{k}$-band state is $\epsilon_{\mathbf{k}}$, while the energy of the atomic configuration with total spin $S$ is given by $E_{S, p[q]}$. The energy difference $E_{\frac{3}{2}, p}-$ $E_{1, q}=\epsilon_{d(p, q)}$ defines the single hole energy level active in the transition between $\left|\frac{3}{2}, M\right\rangle$ and $|1, M-\sigma\rangle_{q}$.

The last term in Eq. $\stackrel{p}{(2)}$ accounts for the interaction between the atom and the surface. This expression is obtained by projecting the fermionic operator $c_{d_{i} \sigma}$, which destroys a hole in the $d_{i}$ orbital, into the selected configuration space:

$$
c_{d_{i} \sigma}=\sum_{M, p, q}|1, M-\sigma\rangle_{q}\left\langle 1, M-\left.\sigma\right|_{q} c_{d_{i} \sigma} \mid \frac{3}{2}, M\right\rangle_{p}\left\langle\frac{3}{2},\left.M\right|_{p} .\right.
$$

Note that only the transitions for which the active orbital is $d(p, q)=d_{i}$ give a nonzero contribution. Equation (3) leads to the following definition of the hopping elements $V_{\mathbf{k} M \sigma}^{p q}$ in Eq. (2) [9]:

$$
V_{\mathbf{k} M \sigma}^{p q}=\left\langle 1, M-\left.\sigma\right|_{q} c_{d(p, q) \sigma} \mid \frac{3}{2}, M\right\rangle_{p} V_{\mathbf{k} d(p, q)} .
$$

Here, $V_{\mathbf{k} d(p, q)}$ is the coupling between the k-band state and the $d(p, q)$ Co orbital. The coefficients $\left\langle 1, M-\left.\sigma\right|_{q} c_{d(p, q) \sigma} \mid \frac{3}{2}, M\right\rangle_{p}$ are calculated by applying the annihilation operator $c_{d(p, q) \sigma}$ to the $|S, M\rangle_{p[q]}$ states expressed in terms of the atomic configurations $\left|d_{1}, d_{2} d_{3}, d_{4} d_{5}\right\rangle$. In this way, we arrive to the expression

$$
\left\langle 1, M-\left.\sigma\right|_{q} c_{d(p, q) \sigma} \mid \frac{3}{2}, M\right\rangle_{p}=p_{p q} \sqrt{\frac{3 / 2+2 \sigma M}{3}} .
$$

In Eq. (5), $p_{p q}$ is $+1(-1)$ if an even (odd) number of permutations are needed to annihilate the hole in the $d(p, q)$ orbital of the configuration $\left|\frac{3}{2}, M\right\rangle_{p}$, and 0 if the transition between $p$ and $q$ is not possible.

\section{Green functions and equations of motion}

We define the following Green functions, which are used for the calculation of the physical quantities of interest [37]:

$$
\begin{gathered}
G_{p q}^{M \sigma}\left(t^{\prime}, t\right)=i \theta\left(t^{\prime}-t\right)\left\langle\left\{\left|\frac{3}{2}, M\right\rangle_{p}\left\langle 1, M-\left.\sigma\right|_{q}\left(t^{\prime}\right), \mid 1, M-\sigma\right\rangle_{q}\left\langle\frac{3}{2},\left.M\right|_{p}(t)\right\}\right\rangle,\right. \\
F_{p q}^{M \sigma}\left(t^{\prime}, t\right)=i\left\langle\left[\left|\frac{3}{2}, M\right\rangle_{p}\left\langle 1, M-\left.\sigma\right|_{q}\left(t^{\prime}\right), \mid 1, M-\sigma\right\rangle_{q}\left\langle\frac{3}{2},\left.M\right|_{p}(t)\right]\right\rangle .\right.
\end{gathered}
$$

Here, $[\cdot, \cdot](\{\cdot, \cdot\})$ is the commutator (anticommutator) and $\langle\cdot\rangle$ is the mean value in the Heisenberg picture. The Green functions (6) and (7) are calculated by using the equations of motion (EOM) method, which is based on the time derivative (in the Heisenberg picture) of the operators that define them. The EOM method requires a suitable decoupling scheme to close the chain of equations of successive Green functions, which involve an increasing number of particles. In this work the EOM are closed in a second order in $V_{\mathbf{k} d_{i}}$, which has been proven to be successful for a qualitative description of the Kondo physics in many atom-surface interacting systems [32,38-40,49].

Due to the degeneracy on the total spin projection $M$, the Green functions become independent of $M$ and $\sigma$, so that we introduce $G_{p q} \equiv G_{p q}^{M \sigma}$ and $F_{p q} \equiv F_{p q}^{M \sigma}$. Assuming that the system has reached the steady-state condition, the Green functions become translationally invariant in time, so that the time dependent equations can be solved by means of 
Fourier transform to obtain the following algebraic system of equations:

$$
\begin{gathered}
\left(\omega-\epsilon_{d(p, q)}-\Sigma_{p q}(\omega)\right) G_{p q}(\omega)=O_{p q}+\Xi_{p q}(\omega), \\
\left(\omega-\epsilon_{d(p, q)}-\Sigma_{p q}^{*}(\omega)\right) F_{p q}(\omega)=\Omega_{p q}(\omega) G_{p q}(\omega)+\Upsilon_{p q}(\omega) .
\end{gathered}
$$

While in equilibrium the physical quantities of interest are obtained from $G_{p q}$, in an out-of-equilibrium situation the Green functions $F_{p q}$ are also needed. In Eqs. (8) and (9), on-site occupations $\left(O_{p q}\right)$ and atom-band crossed terms $\left(\Xi_{p q}\right.$, $\Upsilon_{p q}$ ) are calculated self-consistently together with the Green functions. We focus here on the self-energies $\Sigma_{p q}$ and occupations for the equilibrium situation and refer the reader to Appendix A for the rest of the expressions. The self-energies of Eq. (8) can be expressed as follows:

$$
\begin{aligned}
\Sigma_{p q}(\omega)= & \sum_{q^{\prime} \in p} \Sigma_{d\left(p, q^{\prime}\right)}^{>}\left(\omega+\epsilon_{d\left(p, q^{\prime}\right)}-\epsilon_{d(p, q)}\right) \\
& +\frac{4}{3} \sum_{p^{\prime} \ni q} \Sigma_{d\left(p^{\prime}, q\right)}^{<}\left(\omega+\epsilon_{d\left(p^{\prime}, q\right)}-\epsilon_{d(p, q)}\right) .
\end{aligned}
$$

The first term has contributions from the configurations $q^{\prime}$ to which the state $p$ can fluctuate by losing the hole in the orbital $d\left(p, q^{\prime}\right)$. For example, if $p=d_{1} d_{2} d_{3}$, the three possibilities for $q^{\prime}$ are $d_{2} d_{3}, d_{1} d_{3}$ and $d_{1} d_{2}$, being the active orbital $d\left(p, q^{\prime}\right)=d_{1}, d_{2}$, and $d_{3}$, respectively. This is considered with the restriction $q^{\prime} \in p$ in the sum. Similarly, $p^{\prime} \ni q$ in the second term indicates the contribution of the $p^{\prime}$ states to which $q$ can fluctuate through the capture of a hole in $d\left(p^{\prime}, q\right)$. The $\Sigma_{d_{i}}^{<>>]}$appearing in Eq. (10) are given by

$$
\Sigma_{d_{i}}^{<[>]}(\tilde{\omega})=\int_{-\infty}^{\infty} d \epsilon \frac{f_{<[>]}(\epsilon) \Gamma_{d_{i}}^{0}(\epsilon)}{\tilde{\omega}-\epsilon-i \eta},
$$

where $\Gamma_{d_{i}}^{0}(\epsilon)$ is the imaginary part of the noninteracting selfenergy,

$$
\Sigma_{d_{i}}^{0}(\omega)=\sum_{\mathbf{k}} \frac{\left|V_{\mathbf{k} d_{i}}\right|^{2}}{\omega-\epsilon_{\mathbf{k}}-i \eta} .
$$

In Eq. (11), $\eta$ is the usual infinitesimal convergence parameter, $f_{>}(\epsilon)=1 /\left(e^{(\epsilon-\mu) / k_{\mathrm{B}} T}+1\right)$ is the Fermi function in the hole picture, $f_{<}(\epsilon)=1-f_{>}(\epsilon), \mu$ is the chemical potential, $k_{\mathrm{B}}$ is the Boltzmann constant and $T$ is the temperature. The factors 1 and 4/3 multiplying each term in Eq. (10) come from the summation over $\sigma$ and $M$ of the coefficients given by Eq. (5) (see Appendix A). The self-energies involving couplings with two different orbitals (for instance, $V_{\mathbf{k} d_{1}} V_{\mathbf{k} d_{2}}$ ) are negligible compared with the diagonal ones and are then neglected.

The correlated treatment of the different configurations becomes manifest in Eq. (10), where each self-energy $\Sigma_{p q}$ corresponding to the Green function $G_{p q}$ includes contributions from all the possible virtual fluctuations $p \leftrightarrow q^{\prime}$ and $q \leftrightarrow p^{\prime}$. Let us take the configurations $p=d_{1} d_{2} d_{3}$ and $q=$ $d_{2} d_{3}$ as an example. The fluctuation between them, involving the $d(p, q)=d_{1}$ orbital, incorporates the virtual loss and recapture of all the holes occupying the different orbitals in $p\left(d_{1}, d_{2}\right.$, and $\left.d_{3}\right)$, through the summation over $q^{\prime}$ in Eq. (10).
In the same way, it includes the capture and subsequent loss of holes in the orbitals not present in $q\left(d_{1}, d_{4}\right.$, and $\left.d_{5}\right)$, through the summation over $p^{\prime}$.

The occurrence probabilities of each configuration, indicated by $\left\langle\mid \frac{3}{2}, M\right\rangle_{p}\left\langle\frac{3}{2},\left.M\right|_{p}\right\rangle=\left\langle\frac{3}{2}\right\rangle_{p}$ and $\langle\mid 1, M\rangle_{q}\left\langle 1,\left.M\right|_{q}\right\rangle=$ $\langle 1\rangle_{q}$, are given by

$$
\begin{aligned}
\left\langle\frac{3}{2}\right\rangle_{p} & =\frac{1}{\pi} \int_{-\infty}^{\infty} d \omega f_{<}(\omega) \operatorname{Im} G_{p q}(\omega), \\
\langle 1\rangle_{q} & =\frac{1}{\pi} \int_{-\infty}^{\infty} d \omega f_{>}(\omega) \operatorname{Im} G_{p q}(\omega) .
\end{aligned}
$$

We can construct the one-particle Green function $G_{d_{i} \sigma}\left(t^{\prime}, t\right)=i \theta\left(t^{\prime}-t\right)\left\langle\left\{c_{d_{i} \sigma}^{\dagger}\left(t^{\prime}\right), c_{d_{i} \sigma}(t)\right\}\right\rangle$ by using Eqs. (3) and (5):

$$
G_{d_{i} \sigma}=2 \sum_{p, q} \delta_{d_{i}, d(p, q)} G_{p q} .
$$

The spectral density defined by $G_{d_{i} \sigma}$,

$$
\rho_{d_{i} \sigma}(\omega)=\frac{1}{\pi} \operatorname{Im} G_{d_{i} \sigma}(\omega)
$$

represents the energy distribution of all the fluctuations involving configurations $p$ and $q$ where the orbital $d_{i}$ is occupied in the configuration $p$ and it is empty in the configuration $q$. Then, the mean hole occupation obtained taking into account Eq. (3),

$$
\left\langle c_{d_{i} \sigma}^{\dagger} c_{d_{i} \sigma}\right\rangle=\int_{-\infty}^{\infty} d \omega f_{<}(\omega) \rho_{d_{i} \sigma}(\omega),
$$

gives the contribution to the occupation of the $d_{i}$ orbital provided only by the $S=3 / 2$ configurations. The total hole occupation of the $d_{i}$ orbital, which also includes the contribution from the configurations with $S=1$, is given by

$$
o_{d_{i}}=4 \sum_{p \ni d_{i}}\left\langle\frac{3}{2}\right\rangle_{p}+3 \sum_{q \ni d_{i}}\langle 1\rangle_{q},
$$

where $p \ni d_{i}\left(q \ni d_{i}\right)$ indicates the sum over the configurations $p(q)$ in which $d_{i}$ is occupied, and the sum over $\sigma$ was performed.

\section{Symmetry considerations}

The atomic configurations can be classified according to the symmetry group of the occupied orbitals $(A 1, E 1, E 2)$. For instance, the configurations $p=d_{1} d_{2} d_{4}$ and $p=d_{1} d_{3} d_{4}$ have occupied orbitals with the same symmetry, namely $A 1\left(d_{1}\right)$, $E 1\left(d_{2} / d_{3}\right)$ and $E 2\left(d_{4}\right)$. Then, we have five nonequivalent possibilities for $S=3 / 2$ (labeled with $P$ ) and five for $S=1$ (labeled with $Q$ ), as it is shown in Table II. For each set $P$ $(Q)$ there are $w_{P}\left(w_{Q}\right)$ equivalent configurations degenerated in energy, also indicated in Table II.

The five types of configurations for each value of the total spin $S$ lead to the 11 nonequivalent fluctuations (NEF) shown in Table III. The symmetry of the orbital involved in the fluctuation, $D(P, Q)$, is shown in the same table. $w_{P Q}$ indicates the number of possible equivalent transitions of this type for the active orbital. To illustrate, in the fluctuation between $P=E 1 E 1 E 2$ and $Q=E 1 E 2$, numbered as 2 in Table III, for a given active orbital with symmetry $D=E 1$ there are 
TABLE II. The five nonequivalent possibilities for both, $S=3 / 2$ $(P)$ and $S=1(Q)$, to accommodate the corresponding holes into the three symmetry groups: $E 1, E 2$, and $A 1 . w_{P}\left(w_{Q}\right)$ is the number of equivalent configurations in each set.

\begin{tabular}{lccc}
\hline \hline$P$ & $w_{P}$ & $Q$ & $w_{Q}$ \\
\hline$E 1 E 1 E 2$ & 2 & $E 1 E 1$ & 1 \\
$E 1 E 2 E 2$ & 2 & $E 1 E 2$ & 4 \\
$A 1 E 1 E 1$ & 1 & $E 2 E 2$ & 1 \\
$A 1 E 1 E 2$ & 4 & $A 1 E 1$ & 2 \\
$A 1 E 2 E 2$ & 1 & $A 1 E 2$ & 2 \\
\hline
\end{tabular}

two possibilities for the occupied $E 2$ orbital ( $d_{4}$ or $\left.d_{5}\right)$. Then, $w_{P Q}=2$ in this case.

We should notice that the $11 \mathrm{NEF}$ lead to only 11 nonequivalent $G_{p q}$ in Eq. (8). These nonequivalent Green functions correspond to the fluctuations of Table III and will be identified with $G_{P Q}$. The same notation can be used with the rest of the terms in Eq. (8) $\left(\Sigma_{P Q}, O_{P Q}, \Xi_{P Q}\right)$. In the same way, the energy levels $\epsilon_{d_{i}}$ and the noninteracting self-energies $\Sigma_{d_{i}}^{0}$ depend only on the symmetry group to which the orbital $d_{i}$ belongs, therefore we indicate the three possibilities as $\epsilon_{D}$ and $\Sigma_{D}^{0}$, with $D=E 1, E 2, A 1$. Similarly, the one-particle Green functions defined by Eq. (15) depend only on $D$ and can be expressed in terms of the non equivalent $G_{P Q}$ and their weights $w_{P Q}$ :

$$
G_{D \sigma}=2 \sum_{P, Q} \delta_{D, D(P, Q)} w_{P Q} G_{P Q}
$$

as well as the corresponding spectral densities

$$
\rho_{D}(\omega)=\sum_{\sigma} \frac{1}{\pi} \operatorname{Im} G_{D \sigma}(\omega)
$$

where we sum over $\sigma$ for convenience.

\section{HAMILTONIAN PARAMETERS}

The accuracy of our results relies on an accurate calculation of the different terms that define the proposed ionic Hamiltonian [Eq. (2)]. The first term of Eq. (2) requires the electronic structure of the unperturbed surface, which is calculated by performing a DFT simulation of the graphene without the adatom. In this section, we detail the calculation of the other two terms of Eq. (2): the atom-band coupling terms $V_{\mathbf{k} d_{i}}$, which determine the Hamiltonian parameters $V_{\mathbf{k} M \sigma}^{p q}$ [Eq. (4)], and the total energies $E_{S p[q]}$ of the atomic configurations which lead to the single particle energy levels definition.

\section{A. Atom-band coupling terms}

The self-energies and atom-band crossed terms, which determine the required Green functions in Eqs. (8) and (9), rely on an adequate computation of the coupling terms $V_{n \mathbf{k}} d_{i}$ ( $n$ is the band index, which will be written explicitly in this section).

Our calculation of $V_{n \mathbf{k}} d_{i}$ is based on a linear combination of atomic orbitals (LCAO) expansion of the surface wavefunction $\psi_{n \mathbf{k}}$ [41]. In the LCAO method $\psi_{n \mathbf{k}}$ is expressed in terms of atomic orbitals $\chi_{\alpha}\left(\mathbf{r}-\mathbf{R}_{s}\right)$, corresponding to the $\alpha$ orbital of the $s$ atom of the surface, located in $\mathbf{R}_{s}$. After choosing an adequate Bravais lattice and a base, each atom is univocally located with a basis vector $\mathbf{B}_{b}$ and a lattice vector $\mathbf{L}_{l}, \mathbf{R}_{s}=\mathbf{L}_{l}+\mathbf{B}_{b}$, and for every atom $s$ we can find its basis $b(s)$ and lattice $l(s)$ index. The Bloch theorem leads to the following expression:

$$
\psi_{n \mathbf{k}}(\mathbf{r})=\sum_{\alpha, s} c_{\alpha b(s)}^{n \mathbf{k}} e^{i \mathbf{k} \cdot \mathbf{L}_{l(s)}} \chi_{\alpha}\left(\mathbf{r}-\mathbf{R}_{s}\right),
$$

where the coefficients $c_{\alpha b}^{n \mathbf{k}}$ define the surface density matrix in the symmetrically orthonormalized atomic basis:

$$
\rho_{\alpha \beta s t}(\epsilon)=\sum_{n, \mathbf{k}} e^{i \mathbf{k} \cdot\left(\mathbf{L}_{l(t)}-\mathbf{L}_{l(s)}\right)} c_{\alpha b(s)}^{n \mathbf{k} *} c_{\beta b(t)}^{n \mathbf{k}} \delta\left(\epsilon_{n \mathbf{k}}-\epsilon\right) .
$$

By using the bond-pair model, the $V_{n \mathbf{k} d_{i}}$ couplings are calculated as [41]:

$$
V_{n \mathbf{k} d_{i}}=\sum_{\alpha, s} c_{\alpha b(s)}^{n \mathbf{k}} e^{i \mathbf{k} \cdot \mathbf{L}_{l(s)}} V_{\alpha s d_{i}}
$$

where $V_{\alpha s d_{i}}$ is the coupling between the $\alpha$ orbital of the $s$ surface atom and the $d_{i}$ orbital of the adatom, symmetrically orthogonalized first in the dimeric subspace and then referred to the symmetrically orthogonalized surface atom states [41]. The $V_{\alpha s d_{i}}$ couplings include one and two electron integrals computed within a mean field approximation and using the atomic basis set provided by Huzinaga [50,51].

According to Eq. (23), the square modulus of $V_{n \mathbf{k} d_{i}}$ results

$$
\left|V_{n \mathbf{k} d_{i}}\right|^{2}=\sum_{\alpha, \beta, s, t}\left(e^{i \mathbf{k} \cdot\left(\mathbf{L}_{l(t)}-\mathbf{L}_{l(s)}\right)} c_{\alpha b(s)}^{n \mathbf{k} *} c_{\beta b(t)}^{n \mathbf{k}}\right) V_{\alpha s d_{i}}^{*} V_{\beta t d_{i}}
$$

By using Eqs. (22) and (24), we can evaluate any sum over the indices $n$, $\mathbf{k}$ involving $\left|V_{n \mathbf{k} d_{i}}\right|^{2}$ and a given function $h\left(\epsilon_{n \mathbf{k}}, \ldots\right)$

TABLE III. Nonequivalent fluctuations between the five sets of configurations with three holes $(\mathrm{P})$ and the five with two holes $(\mathrm{Q})$ of the Co orbitals splitted into the three symmetry groups. The symmetry of the active orbital involved in the transition is indicated by $D(P, Q)$ and the number of equivalent transitions for the active orbital by $w_{P Q}$.

\begin{tabular}{lccccccccccc}
\hline \hline Fluctuation & 1 & 2 & 3 & 4 & 5 & 6 & 7 & 8 & 9 & 10 \\
\hline$P$ & $E 1 E 1 E 2$ & $E 1 E 1 E 2$ & $E 1 E 2 E 2$ & $E 1 E 2 E 2$ & $A 1 E 1 E 1$ & $A 1 E 1 E 1$ & $A 1 E 1 E 2$ & $A 1 E 1 E 2$ & $A 1 E 1 E 2$ & $A 1 E 2 E 2$ & $A 1 E 2 E 2$ \\
$Q$ & $E 1 E 1$ & $E 1 E 2$ & $E 1 E 2$ & $E 2 E 2$ & $E 1 E 1$ & $A 1 E 1$ & $A 1 E 1$ & $E 1 E 2$ & $A 1 E 2$ & $A 1 E 2$ & $E 2 E 2$ \\
$D(P, Q)$ & $E 2$ & $E 1$ & $E 2$ & $E 1$ & $A 1$ & $E 1$ & $E 2$ & $A 1$ & $E 1$ & $E 2$ & $A 1$ \\
$w_{P Q}$ & 1 & 2 & 2 & 1 & 1 & 1 & 2 & 4 & 2 & 1 \\
\hline \hline
\end{tabular}




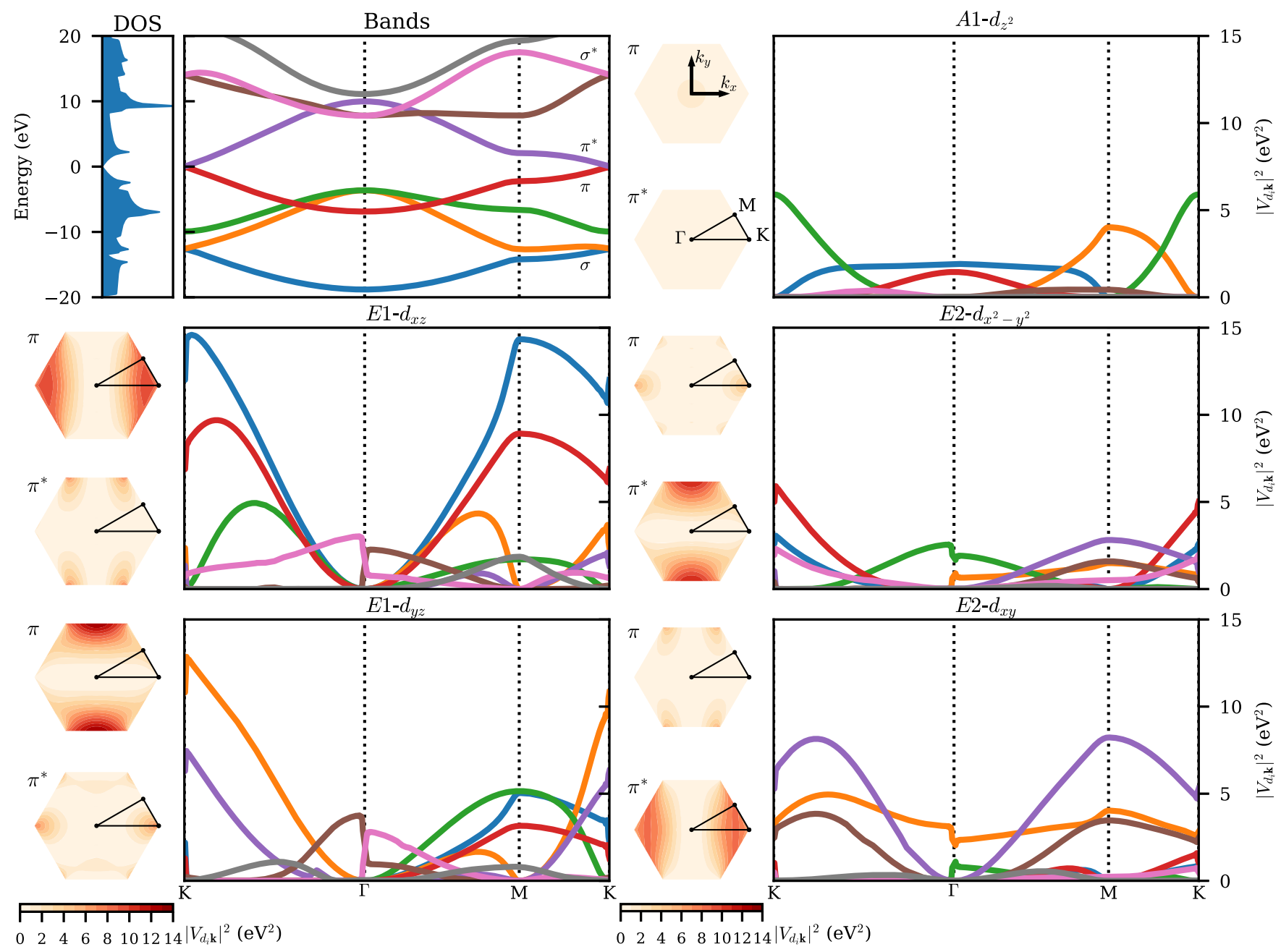

FIG. 1. DOS and band structure of pure graphene (upper left) and square modulus of the atom-band couplings $\left|V_{n \mathbf{k} d_{i}}\right|^{2}$ for the five $d$ orbitals of Co at the equilibrium distance on the hollow position. The colors show the correspondence between the graphene bands $n$ and the $\left|V_{n \mathbf{k}} d_{i}\right|^{2}$. The contour plots show the $\left|V_{n \mathbf{k} d_{i}}\right|^{2}$ for each orbital for the $\pi$ and $\pi^{*}$ bands. The $\mathbf{k}$ path followed in the plots (KГMK) is indicated in the contour plots. The jumps observed at the $K$ point for the $\pi$ and $\pi^{*}$ bands and at the $\Gamma$ point for some $\sigma$ bands are not numerical artifacts and they correspond to an accumulation of contour lines in the contour plots.

as follows:

$$
\begin{aligned}
& \sum_{n, \mathbf{k}}\left|V_{n \mathbf{k} d_{i}}\right|^{2} h\left(\epsilon_{n \mathbf{k}}, \ldots\right) \\
& \quad=\sum_{\alpha, \beta, s, t} V_{\alpha s d_{i}}^{*} V_{\beta t d_{i}} \times \int_{-\infty}^{\infty} d \epsilon \rho_{\alpha \beta s t}(\epsilon) h(\epsilon, \ldots) .
\end{aligned}
$$

For $\Sigma_{d_{i}}^{0}$ in Eq. (12), $h\left(\epsilon_{n \mathbf{k}}, \ldots\right)=1 /\left(\omega-\epsilon_{n \mathbf{k}}-i \eta\right)$.

Note that by using Eq. (25), we build the self-energies and other quantities related to $V_{n \mathbf{k} d_{i}}$ from the density matrix of the surface without the impurity, avoiding supercell effects that are present in other methods [12,14]. Also, we use a graphene primitive cell to calculate the coefficients $c_{\alpha b}^{n \mathbf{k}}$, which allows us to keep track of the $n \mathbf{k}$ state to which each impurity orbital is coupled [Eq. (24)].

In Fig. 1, we show the $\left|V_{n \mathbf{k}} d_{i}\right|^{2}$ quantities computed using Eq. (24) for each $d_{i}$ Co orbital, in correspondence with the graphene bands and for the equilibrium distance $(1.52 \AA)$ of the Co atom adsorbed on the hollow position. The contour plots show the $\left|V_{n \mathbf{k} d_{i}}\right|^{2}$ corresponding to the $\pi$ and $\pi^{*}$ bands in the first Brillouin zone. It becomes evident the small coupling of the $A 1$ group with the graphene bands, which is larger for low-lying bands. The antisymmetry between the $E 1$ and $E 2$ groups is remarkable [8,12]: while the $E 1$ group strongly couples with the $\pi$ band and almost nothing with the $\pi^{*}$, the behavior of the orbitals of the $E 2$ group is precisely the inverse, with similar magnitudes. Notice that, despite the symmetry shared by the orbitals in the $E 1$ (E2) group, each orbital couples with different regions of the first Brillouin zone. In particular, each orbital of the $E 1$ (E2) group presents a strong coupling with the $\pi\left(\pi^{*}\right)$ band in different $M$ points, where the van Hove singularities are located.

By using Eqs. (12) and (25), we obtain the noninteracting self-energies $\Sigma_{D}^{0}$. Figure 2 shows the real $\left(\Lambda_{D}^{0}\right)$ and imaginary $\left(\Gamma_{D}^{0}\right)$ parts of $\Sigma_{D}^{0}$ for each symmetry group. In panels (a) and (b), we compare the obtained Anderson width $\Gamma_{D}^{0}$ with results from previous works $[11,12]$ for the same system. An overall agreement is observed. However, notice that our calculation leads to a smooth $\Gamma_{D}^{0}$, like the one obtained in Ref. [12] by using a tight-binding approximation. The somehow noisy 


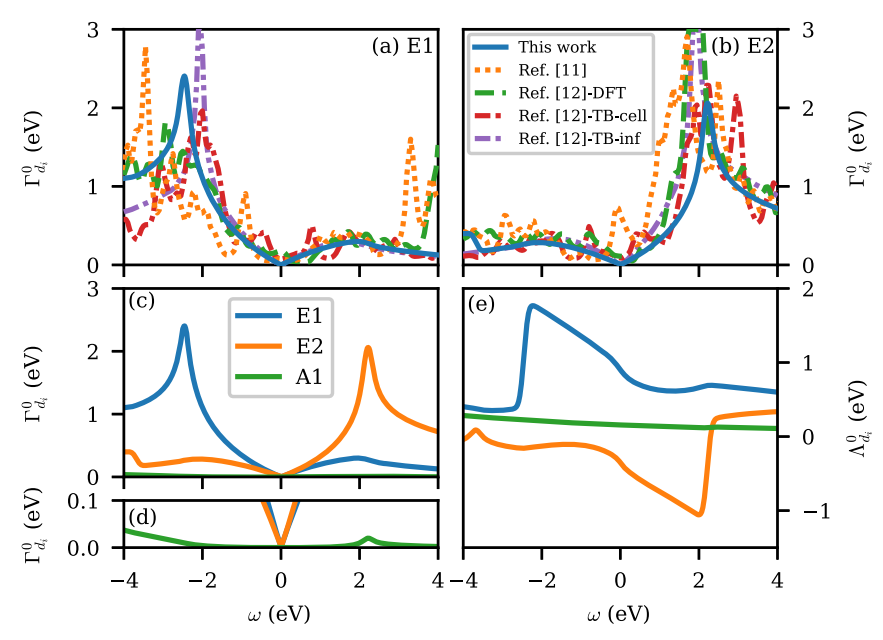

FIG. 2. Our calculated $\Gamma_{d}^{0}$ compared with those from Refs. [11,12] for (a) $E 1$ and (b) $E 2$ groups. (c) Presents comparatively our calculated $\Gamma_{d}^{0}$ for the three symmetry groups, (d) is a zoom and (e) corresponds to the real part of the noninteracting self-energy, $\Lambda_{d}^{0}$.

behavior of the other calculations is attributed to supercell effects [12], which are absent in our approach. The preferential coupling of the $E 1$ (E2) group with the $\pi\left(\pi^{*}\right)$ band is expressed through an asymmetry of the $\Gamma_{D}^{0}$ for each group: the coupling is stronger at $\omega<0 \mathrm{eV}(\omega>0 \mathrm{eV})$, with peaks related to the van Hove singularities in the $\mathrm{M}$ points of the first Brillouin zone [12].

For the calculated equilibrium distance, both $\Gamma_{E 1}^{0}$ and $\Gamma_{E 2}^{0}$ have the same slope when $\omega \rightarrow 0$. Moreover, both $\Gamma_{D}^{0}$ are practically specular images around $\omega=0$, that is $\Gamma_{E 1}^{0}(\omega) \approx$ $\Gamma_{E 2}^{0}(-\omega)$. This is caused by the similar values of the atomic hoppings $V_{\alpha s d_{i}}$ for the $E 1$ and $E 2$ orbitals at the equilibrium distance of $1.52 \AA$. When the Co-graphene distance is increased, the relative height of the the $E 1$ peak with respect to the $E 2$ one increases, since the coupling is more favorable for this symmetry (the $E 1$ orbitals are located out of the $x y$ plane). The opposite occurs if the atom-surface distance is smaller than $1.52 \AA$ : in this case, the coupling of the $E 2$ orbitals, located on the $x y$ plane, becomes more favorable and the peak in $\Gamma_{E 2}^{0}$ grows with respect to the one in $\Gamma_{E 1}^{0}$. Although each orbital of both groups, $E 1$ and $E 2$, couples differently with the respective band (see Fig. 1), the resulting $\Gamma_{D}^{0}$ depends only on the symmetry group. Nevertheless, the underlying different couplings $V_{n \mathbf{k} d_{i}}$ could be still probed in systems with these peculiarities by, for instance, azimuthal angle dependent ion scattering spectroscopy [52].

The real part of the noninteracting self-energy $\Lambda_{D}^{0}$ [Fig. 2(e)] will shift the $\epsilon_{E 1}$ level to higher energies, the $\epsilon_{E 2}$ level to lower energies and will have almost no effect on $\epsilon_{A 1}$. This effect is further discussed in the next section.

\section{B. Energy levels}

The computation of the orbital energies $\epsilon_{D}$ is a challenging task and usually involves several approximations. The energy levels are generally estimated from DFT calculations, which requires to account for double counting corrections of difficult determination $[8,11,12,53]$. In addition,
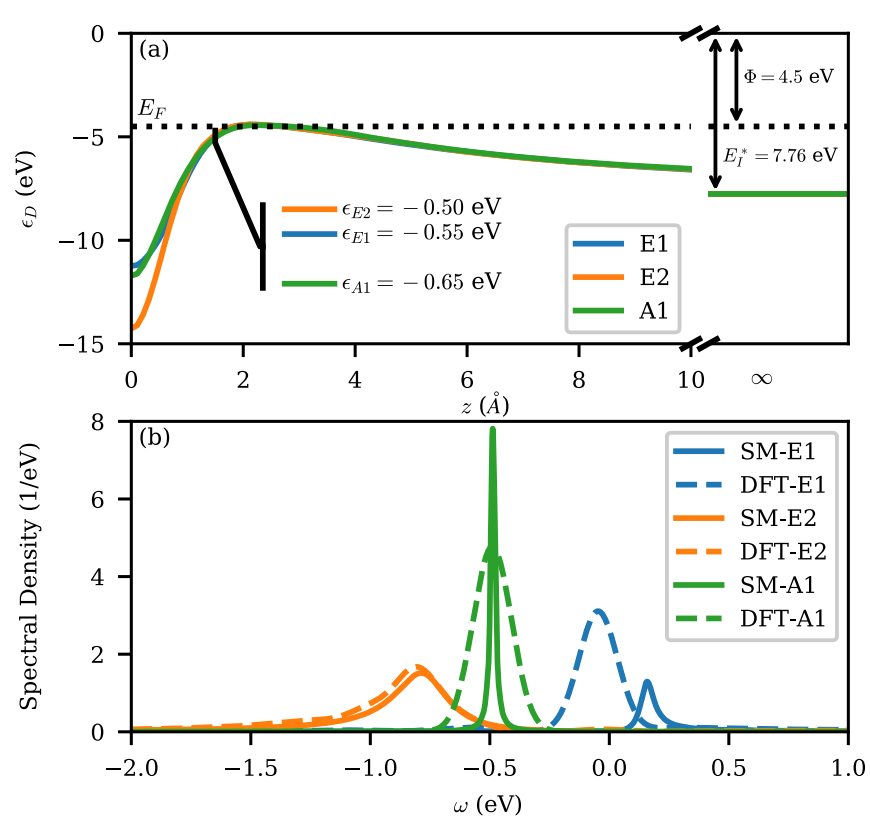

FIG. 3. (a) Energy levels as a function of the Co-graphene distance $z$ for each group, shown relative to the vacuum. The asymptotic value $E_{I}^{*}$ and the work function of graphene $\Phi$ are indicated. The detail at the Co equilibrium position (1.52 $\AA$ ) shows the level positions with respect to the graphene Fermi level $\left(E_{F}\right)$. (b) Co LDOS obtained with DFT compared with the results of the simplified model (SM) described in the text.

the noninteracting system solved by the DFT method in principle do not give reliable energy eigenstates for the real system [54].

We obtain the one electron energy levels corresponding to the transitions occurring in the presence of the surface as the the difference between the total energies of the atomic configurations involved, $\epsilon_{d(p, q)}=E_{\frac{3}{2}, p}-E_{1, q}$. The total energies $E_{S, p[q]}$ are calculated from a mean field approximation of the many-body Hamiltonian that describes the atom-surface interaction, by considering a second order expansion in the atomic overlap of the symmetrically orthogonalized basis set [41] (see Appendix B).

In the calculation of the total energies, we take into account the electron that is transferred from the Co atom to the graphene surface in the equilibrium configuration, by distributing it among the $72 \mathrm{C}$ atoms of the supercell $(\approx 0.014$ electrons per $\mathrm{C}$ atom). The long range interactions are also included by means of the image potential $1 / 4 z$, which defines the dependence of the energy levels with the normal distance to the surface $z$ (in a.u.), for distances larger than $z_{c}=4 \AA$ $[55,56]$.

Far from the surface, the ground state of the Co atom corresponds to the electronic configuration $3 d^{7} 4 s^{2}$. At the equilibrium position, our DFT calculations suggest that the configuration fluctuates between $3 d^{7}$ and $3 d^{8}$, with only one electron distributed in the $s$ and $p$ orbitals. In order to approximate the situation near the surface we refer the energy levels to an asymptotic value calculated from the measured energies of the excited neutral $\left(3 d^{8} 4 s^{1}\right)$ and ionic $\left(3 d^{7} 4 s^{1}\right)$ configurations, $E_{I}^{*}=E\left(3 d^{8} 4 s^{1}\right)-E\left(3 d^{7} 4 s^{1}\right)=7.76 \mathrm{eV}$ [57] (see Fig. 3). 
Finally, the energy levels are measured with respect to the graphene Fermi level $\left(E_{F}\right)$, by assuming a work function $\Phi=$ $4.5 \mathrm{eV}[58,59]$. The resulting $\epsilon_{D}$ for each symmetry group as a function of the Co-graphene distance are shown in Fig. 3(a).

Clearly, a variation of either the matching distance of the image potential $z_{c}$ or the graphene work function $\Phi$ introduces changes in the absolute value of the energy levels, although these variations do not affect the splitting between the levels predicted by our calculation. In order to check the reliability of the obtained energy values, we computed the local spectral density in the Co atom by using a simplified model which disregards the correlation among the configurations introduced via the interaction with the band states. This result is obtained by keeping only the terms with $q^{\prime}=q$ and $p^{\prime}=p$ in Eq. (10), and doing the same in the expression for $\boldsymbol{\Xi}_{p q}$ [see Eq. (A15)]. The spectral density computed by using this model is then compared with the result obtained from the DFT calculation in Fig. 3(b).

The $E 2$ and $A 1$ spectral densities show resonances that are in good agreement with the DFT results. On the other hand, the $E 1$ resonance, located at $\omega \approx 0 \mathrm{eV}$ in the DFT LDOS, is shifted towards higher energies when using our simplified calculation. The position of the $E 1$ resonance is related to the shift produced by the real part of the self-energy $\Lambda_{E 1}^{0}$ [see Fig. 2(e)]. The marked decrease of $\Lambda_{E 1}^{0}(\omega)$ in the range $-2 \mathrm{eV} \lesssim \omega \lesssim 0 \mathrm{eV}$ leads to a small variation of the $E 1$ resonance position in this range of $\omega$ values. Then, an overall good agreement is obtained between the simplified model and the DFT results, supporting the proposed calculation of the energy levels. Different proposals in this direction have been developed to obtain the energy levels, e.g., by matching the result from a Hartree-Fock approximation of the Anderson Hamiltonian to the DFT LDOS [53].

In summary, we take as reference values for the Co energy levels $\epsilon_{E 1}=-0.50 \mathrm{eV}, \epsilon_{E 2}=-0.55 \mathrm{eV}$, and $\epsilon_{A 1}=-0.65 \mathrm{eV}$, measured with respect to the graphene Fermi level. Nevertheless, we will also analyze the effect of rigid variations of these reference energy levels on the physical quantities of interest.

\section{CO-GRAPHENE SYSTEM IN EQUILIBRIUM}

\section{A. Occurrence probabilities and spectral densities}

The proposed approach allows to identify the weight of each atomic configuration in the ground state of the interacting system. In Figs. 4(a) and 4(b), we show the occurrence probability of each set of equivalent configurations $P$ and $Q$ of Table II, calculated in the following way:

$$
\begin{aligned}
\left\langle\frac{3}{2}\right\rangle_{P} & =\sum_{M, p \in P}\left\langle\mid \frac{3}{2}, M\right\rangle_{p}\left\langle\frac{3}{2},\left.M\right|_{p}\right\rangle, \\
\langle 1\rangle_{Q} & =\sum_{M, q \in Q}\langle\mid 1, M\rangle_{q}\left\langle 1,\left.M\right|_{q}\right\rangle .
\end{aligned}
$$

In Eq. (26), we sum over the $w_{P}$ equivalent configurations of the $P$ set and the four equivalent $M$ possibilities for $S=$ $3 / 2$. Similarly, in Eq. (27) the sum is over the $w_{Q}$ equivalent configurations of the $Q$ set and the three projections of $S=1$.

In Fig. 4(c), we present the total hole occupation for each symmetry group, calculated by using Eq. (18). To facilitate the analysis, panel (d) shows the shifted energy levels in the

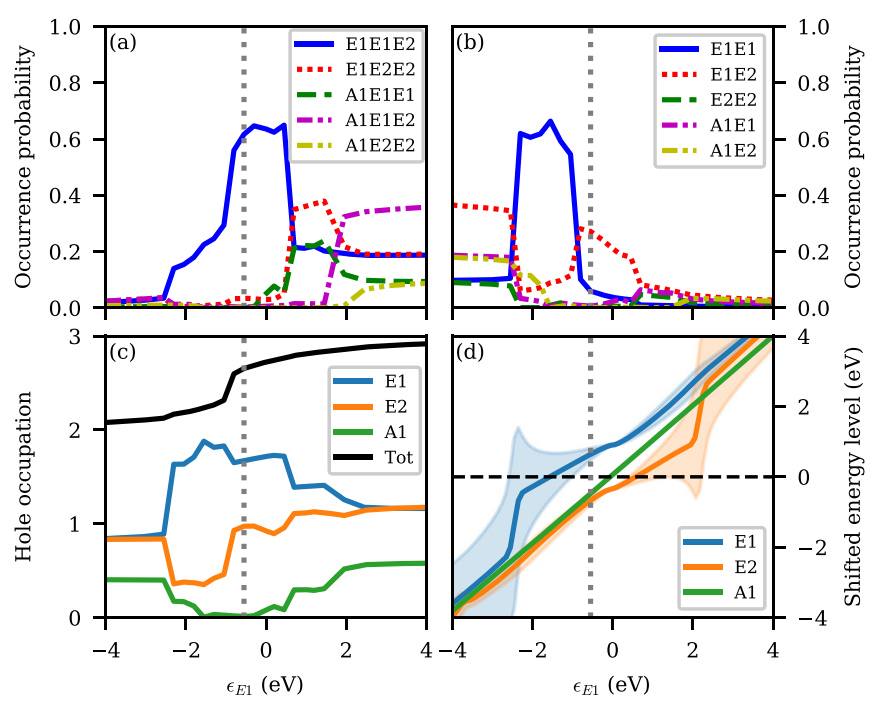

FIG. 4. Occurrence probabilities for the configurations with (a) three and (b) two holes. (c) Hole occupation per symmetry group. (d) Shifted energy levels $\epsilon_{D}+\Lambda_{D}^{0}\left(\epsilon_{D}\right)$ for the noninteracting case, broadened by $\Gamma_{D}^{0}\left(\epsilon_{D}\right)$. The results are shown as a function of the energy level position $\epsilon_{E 1}$ after shifting rigidly the reference levels $\epsilon_{E 1}=-0.55 \mathrm{eV}$ (indicated by a dotted line), $\epsilon_{E 2}=-0.50 \mathrm{eV}$, and $\epsilon_{A 1}=-0.65 \mathrm{eV}$.

noninteracting case, $\epsilon_{D}^{S}=\epsilon_{D}+\Lambda_{D}^{0}\left(\epsilon_{D}\right)$, broadened by $\Gamma_{D}^{0}\left(\epsilon_{D}\right)$. The results are presented as a function of the $\epsilon_{E 1}$ energy level position, used to indicate a rigid shift of the energy levels with respect to the reference values $\epsilon_{E 1}=-0.55 \mathrm{eV}, \epsilon_{E 2}=-0.50 \mathrm{eV}$, and $\epsilon_{A 1}=-0.65 \mathrm{eV}$ calculated in Sec. IV B.

When the broadened energy levels are well below the Fermi level, the probability of occurrence of the states with three holes is practically zero [Fig. 4(a)], and only the configurations with two holes are occupied [Fig. 4(b)]. In this situation, the splitting between the energy levels is not relevant and all the two holes configurations have the same probability of occurrence. The different values in Fig. 4(b) are due to the different $w_{Q}$ equivalent configurations in each $Q$ set [Eq. (27)].

When $\epsilon_{E 1} \approx-2.5 \mathrm{eV}$ there is an abrupt increase in the occurrence probability of the $E 1 E 1$ configuration [Fig. 4(b)]. This change corresponds to the sudden increase in $\Lambda_{E 1}^{0}$ [Fig. 2(e)] which produces a jump in the shifted energy level $\epsilon_{E 1}^{S}$ [Fig. 4(d)]. The $\epsilon_{E 1}^{S}$ level is then clearly separated from the other two levels and situated closer to $E_{F}$, increasing in this way the probability of being occupied by holes [Fig. 4(c)].

The next abrupt change in the $E 1 E 1$ occurrence probability, at $\epsilon_{E 1} \approx-1 \mathrm{eV}$, corresponds to the broadened energy level $\epsilon_{E 1}^{S}$ crossing the Fermi level, as it is seen in panel (d). At this single hole energy the configuration $E 1 E 1 E 2$ becomes the dominant one. The width of the $E 2$ level allows fluctuations involving this orbital, while the two $E 1$ orbitals remain preferentially occupied by holes. As a consequence, the hole occupation of $E 2$ [panel (c)] presents a sudden increase that is responsible for the change observed in the total occupation shown in the same panel. This behavior, also observed in Ref. [8], is a signal of a transition to a ground state with 
a predominant weight of the $S=3 / 2$ configurations, being $E 1 E 1 E 2$ the most probable. The active orbital involved in this transition belongs to the $E 2$ symmetry group.

At $\epsilon_{E 1} \gtrsim 1 \mathrm{eV}$, all the energy levels are well defined above the Fermi level and the occurrence probabilities tend to an equal value for all the configurations with three holes. As before, the different values are only due to the different number of equivalent configurations $w_{P}$ in each $P$ set [Eq. (26)].

The value $\epsilon_{E 1}=-0.55 \mathrm{eV}$, indicated with a dotted line in Fig. 4, corresponds to the reference energy levels. For these levels, the corresponding shifted values are $\epsilon_{E 1}^{S}=0.64 \mathrm{eV}$, $\epsilon_{E 2}^{S}=-0.67 \mathrm{eV}$ and $\epsilon_{A 1}^{S}=-0.48 \mathrm{eV}$. The shifts caused by $\Lambda_{D}^{0}$ change the ordering of the levels to $\epsilon_{E 2}^{S}<\epsilon_{A 1}^{S}<$ $\epsilon_{E 1}^{S}$, which is consistent with previous results $[8,12]$. From Fig. 4(a), we observe that the three-hole set $E 1 E 1 E 2$ is the most probable at the reference energy levels, with an occurrence probability of 0.62 . However, there is also an important contribution (0.35) from the two-hole state $E 1 E 2$ (panel (b)). This mixture of configurations with $S=3 / 2$ and 1 clearly corresponds to a mixed-valence regime. The electron occupation at the reference energy levels for the $E 1, E 2$, and $A 1$ groups, derived from Fig. 4(c), correspond to 2.32, 3.04, and 1.98, respectively. These values are consistent with our calculated DFT occupations presented in Sec. II and also with previous results $[8,11]$.

In Fig. 5, we present the spectral densities $\rho_{D}$ calculated from Eq. (20), for each symmetry group and at different energy level positions. The possible fluctuations involving the active orbital $D$ are contained in the Green functions $G_{P Q}$ with $D(P, Q)=D$ (Table III). The positions and widths of the peaks corresponding to each $G_{P Q}$ are determined by the self-energies $\Sigma_{P Q}$ defined by Eq. (10). Each $\Sigma_{P Q}$ contains contributions from the different orbitals, with weights that depend on the involved configurations $P$ and $Q$. The involved virtual transitions generate structures in the spectral densities, related to the presence of electronic correlation in a multiorbital system $[43,60]$. The dominant fluctuations that contribute to the spectral densities $\rho_{D}$ are indicated with labels in Fig. 5, showing the three and two holes configurations, $P$ and $Q$, that fluctuate. Spectral weight at $\omega>0 \mathrm{eV}$ indicates the occurrence probability of the three holes configurations $P$ with the active orbital occupied, while at $\omega<0 \mathrm{eV}$ it gives the probability of the two holes configurations $Q$ with the active orbital empty.

We start our analysis at the reference energy level positions, corresponding to $\epsilon_{E 1}=-0.55 \mathrm{eV}$. The main contribution to $\rho_{E 1}$ in Fig. 5(a) is given by the fluctuation between the configurations with the highest occurrence probabilities, namely, $E 1 E 1 E 2$ and $E 1 E 2$ [Figs. 4(a) and 4(b)], being $E 1$ the active orbital. In a similar way, the fluctuation between $E 1 E 1 E 2$ and $E 1 E 1$ is the main contribution to $\rho_{E 2}$ [panel (b)]. These three configurations ( $E 1 E 1 E 2, E 1 E 2$, and $E 1 E 1)$ represent around $95 \%$ of the total occurrence probability, and therefore the fluctuations corresponding to the transitions between these states dominate the spectral densities. Both resonancelike peaks occur at $\omega>0 \mathrm{eV}$, indicating that the three holes configuration $E 1 E 1 E 2$ is the most probable.

In the case of $\rho_{A 1}$ [Fig. 5(c)], there is a small contribution to the spectral density that arises from the fluctuation between

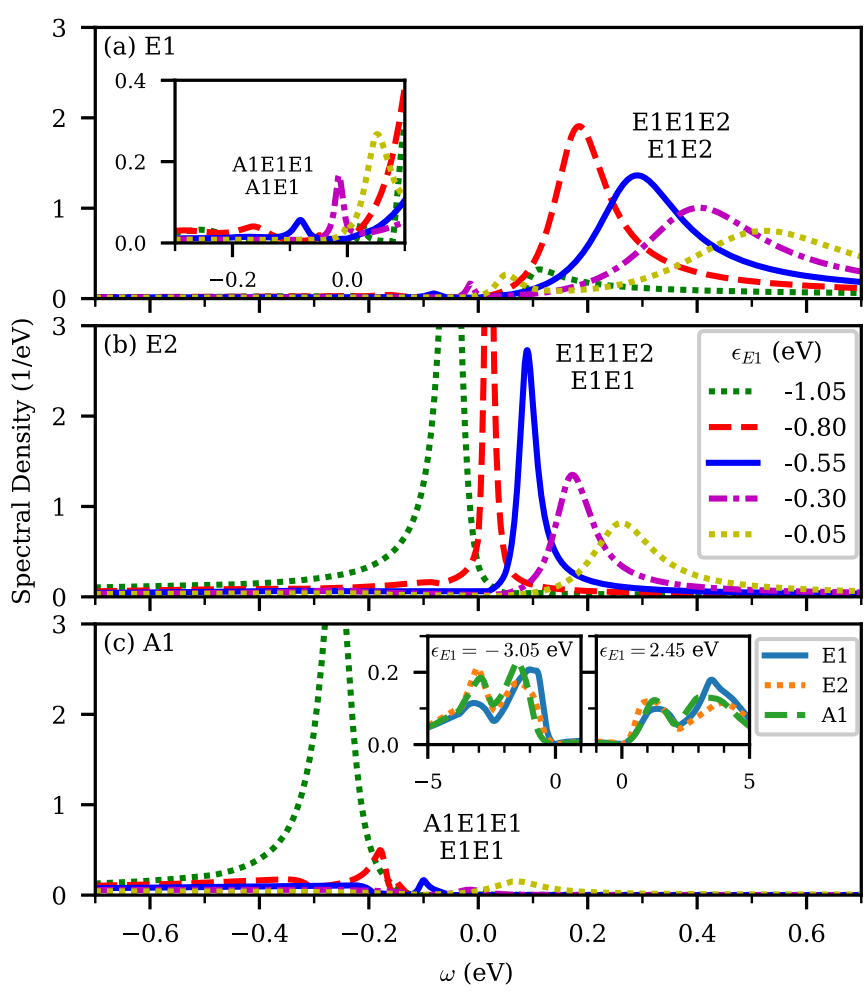

FIG. 5. Spectral densities $\rho_{D}$ calculated at several values of the energy levels, keeping constant their splitting and indicating their positions with the value of $\epsilon_{E 1}$. The main resonance in each case is associated to the fluctuation between the three and two holes configurations labeled with $P$ (upper line) and $Q$ (lower line). The inset in (a) shows a zoom near zero, where other fluctuations of lower probability can be observed. The insets in (c) show the spectral densities for the levels well below and well above the Fermi level.

the configurations $E 1 E 1$ and $A 1 E 1 E 1$. Given that $\Gamma_{A 1}^{0} \approx$ $0 \mathrm{eV}$ near the Fermi level, this low probability transition is only possible due to the interaction between the $d$ orbitals mediated by the graphene bands. The $A 1 E 1 E 1$ configuration also introduces a small contribution to $\rho_{E 1}$, related to the fluctuation to the $A 1 E 1$ state [inset of Fig. 5(a)]. These small contributions are enhanced as the occurrence probability of the $A 1 E 1 E 1$ configuration increases, which occurs when the energy levels are shifted towards higher energies [Fig. 4(a)], or when the temperature is increased (see Sec. V C).

When the levels are shifted towards lower energies $\left(\epsilon_{E 1}=\right.$ $-1.05 \mathrm{eV})$, the main peak observed in $\rho_{E 1}$ tends to disappear, while the one in $\rho_{A 1}$ increases [Figs. 5(a) and 5(c)]. This occurs in coincidence with the change of the preferential configuration, from $E 1 E 1 E 2$ to $E 1 E 1$ (Fig. 4). The vanishing $E 1$ peak has been related to a decrease in the interaction width $\Gamma_{E 1}^{0}(\omega)$ when $\omega<0 \mathrm{eV}$ [8], but $\Gamma_{E 1}^{0}$ actually increases in this region [Fig. 2(c)]. Our calculations suggest instead the following interpretation. When $-2.5 \mathrm{eV} \lesssim \epsilon_{E 1} \lesssim-1.0 \mathrm{eV}$, a $S=1$ state dominates, being the two holes configuration $E 1 E 1$ the most stable [Fig. 4(b)]. Then, the most important fluctuations involve the capture of a hole in the $E 1 E 1$ configuration, being either $A 1$ or $E 2$ the active orbitals, but not $E 1$, which is already completely occupied. The peaks in $\rho_{E 2}$ and $\rho_{A 1}$ at $\omega<0 \mathrm{eV}$ indicate precisely these transitions, between $E 1 E 1$ 


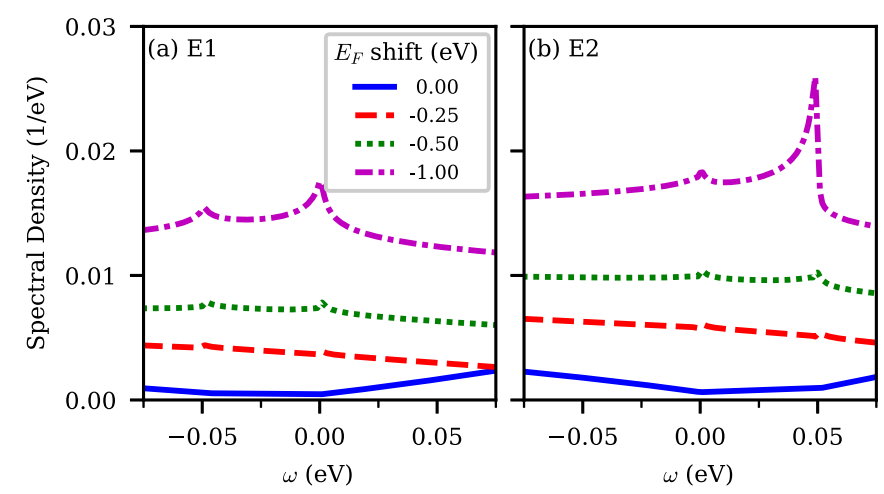

FIG. 6. Spectral densities $\rho_{D}$ calculated at several Fermi level shifts. The energy levels are maintained at the position corresponding to $\epsilon_{E 1}=2.45 \mathrm{eV}$ from the Fermi level in all cases.

and $E 1 E 1 E 2$ or $A 1 E 1 E 1$. The fact that the peak in $\rho_{E 1}$ do not cross $\omega=0 \mathrm{eV}$ when lowering the energy levels indicates that the three hole configurations $E 1 E 1 E 2$ is more favorable than $E 1 E 2$, since the weight of the former is enhanced by the virtual fluctuations of the most probable configuration $E 1 E 1$. To summarize, at $\epsilon_{E 1}=-1.05 \mathrm{eV}$, only the orbitals other than $E 1$ give place to appreciable peaks in the spectral density at $\omega<0 \mathrm{eV}$, since the capture of a hole is only possible in those orbitals.

If the energy levels are positioned well below or well above the Fermi level, the orbitals become practically degenerated and their energy widths become comparable. Then, the spectral densities for the three groups are similar and the spectral weight is only significant either below or above the Fermi level, indicating a ground state with a well defined total spin for the Co adatom, $S=1$ or $S=3 / 2$, respectively [insets of Fig. 5(c)].

The spin fluctuation $S$ to $S-1 / 2$ in the adsorbed atom is related to an antiferromagnetic interaction with the surface when the spin $S$ corresponds to the ground state, and in this case a Kondo resonance is expected [61]. The absence of the Kondo structure in the spectral densities for the energy level positions corresponding to $\epsilon_{E 1}=2.45 \mathrm{eV}$ [inset of Fig. 5(c)] is due to the null density of states of graphene at the Fermi level. This situation is modified in doped graphene, where $E_{F}$ is shifted with respect to the Dirac point. In Fig. 6, we present the spectral densities $\rho_{E 1}$ and $\rho_{E 2}$ for several shifts of $E_{F}$ and by keeping the one particle energy levels in the position corresponding to $\epsilon_{E 1}=2.45 \mathrm{eV}$ with respect to $E_{F}$. We observe that a Kondo peak begins to emerge in both $\rho_{E 1}$ and $\rho_{E 2}$ when the Fermi level is $0.5 \mathrm{eV}$ below the Dirac point. Note that $\rho_{E 1}\left(\rho_{E 2}\right)$ presents a second peak at $-0.05 \mathrm{eV}$ $(0.05 \mathrm{eV})$, introduced by the self-energies [Eq. (10)] at the difference of the energy levels $\epsilon_{E 1}=2.45 \mathrm{eV}$ and $\epsilon_{E 2}=$ $2.50 \mathrm{eV}$. Even in this condition, no Kondo peak is expected in $\rho_{A 1}$, due to the negligible coupling of the $A 1$ orbital with graphene.

\section{B. Approximations by reducing the configuration space}

We will analyze two further approximations obtained by restricting the space of configurations and, consequently, the involved fluctuations. Given that the DFT calculations show
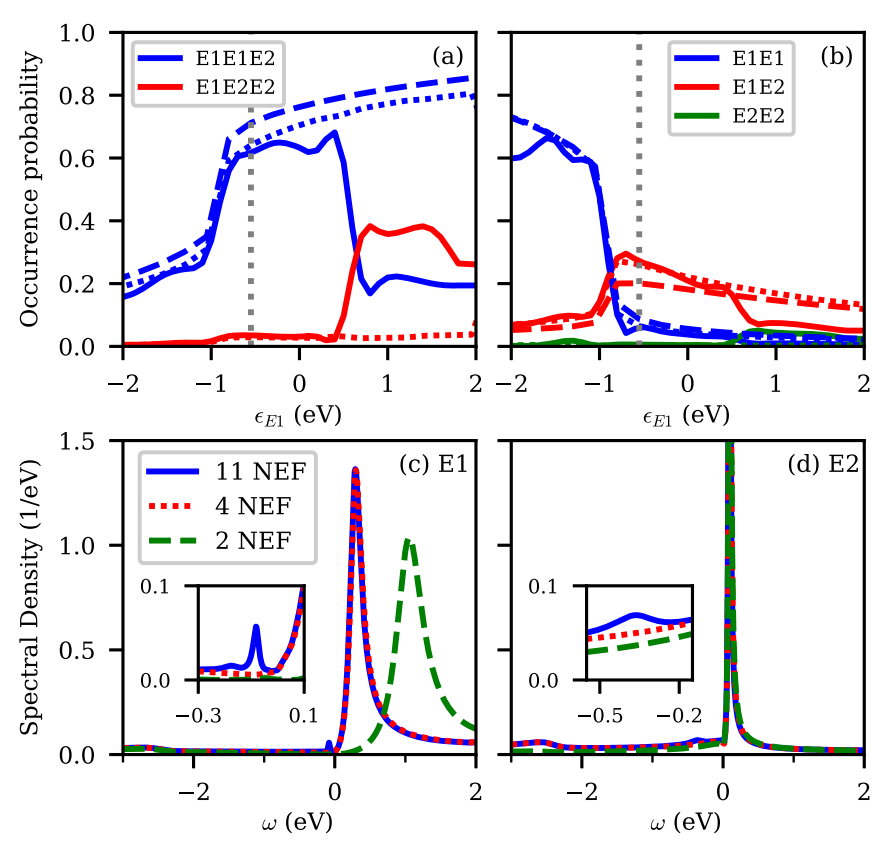

FIG. 7. Occurrence probabilities for the configurations with (a) $S=3 / 2$ and (b) $S=1$, calculated by including $11 \mathrm{NEF}$ (full line), $4 \mathrm{NEF}$ (dotted line), and $2 \mathrm{NEF}$ (dashed line) as a function of the energy level positions. Spectral densities obtained from each approximation for (c) $E 1$ and (d) $E 2$ orbitals, at the reference energy levels (shown with a doted line in the upper panels). The insets show a zoom near zero, where structures appearing in the 11 NEF case can be appreciated.

that the $A 1$ orbital is nearly completely empty of holes, restricting the space to the configurations in which this orbital is empty should be a reasonable approximation. In this case, the space is given by 4 NEF (1-4 in Table III). As a second approximation, and considering that the DFT calculations suggest a hole occupation in the $E 2$ orbitals between 0 and 1 , we keep the configurations with up to one hole in these orbitals, which gives two possible NEF (1 and 2 in Table III). Then, we have three levels of approximation: the complete calculation with $11 \mathrm{NEF}$, the calculation including only the configurations without holes in $A 1$ (4 NEF) and finally the one restricted to the configurations with no holes in $A 1$ and up to one hole in $E 2$ ( $2 \mathrm{NEF}$ ).

Since the 2 and 4 NEF approximations assume that $A 1$ is empty of holes, they are expected to reproduce the results of the total space (11 NEF) in the region where $A 1$ has a very low occupation, that is the range $-2.0 \mathrm{eV} \lesssim \epsilon_{E 1} \lesssim 0.5 \mathrm{eV}$ [see Fig. 4(c)]. In Fig. 7, we compare the occurrence probabilities calculated by using the three approximations as a function of the energy level positions, and also the spectral densities at the reference energy levels.

As expected, we observe a good agreement between the three approximations in the range where $A 1$ is completely empty of holes. Above $\epsilon_{E 1} \approx 0.5 \mathrm{eV}$ the 2 and 4 NEF approximations predict a similar occurrence probability of the $E 1 E 1 E 2$ configuration, which is higher than the probability obtained with $11 \mathrm{NEF}$. Around the reference energy levels $\left(\epsilon_{E 1}=-0.55 \mathrm{eV}\right)$, the $4 \mathrm{NEF}$ calculation provides a good 
description of the orbital occupations, compared with the 11 NEF results.

In Figs. 7(c) and 7(d), we can see that, although the occurrence probabilities are similar for the three approximations at the reference energy levels, the spectral densities show quite important differences. First, the calculation with the complete subspace (11 NEF) takes into account the $A 1$ orbital and therefore it allows to calculate the associated spectral density $\rho_{A 1}$. Also, the peak in $\rho_{E 1}$, related to the fluctuation between the configurations $A 1 E 1 E 1$ and $A 1 E 1$ for the 11 NEF calculation [inset of Fig. 7(c)], is absent in the other approximations where $A 1$ is ignored. Then, an important feature of the $\rho_{E 1}$ spectral density around the Fermi level is lost when the $A 1$ orbital is eliminated from the calculation. Even when the $A 1$ occupation is negligible, including the possibility of occupation of this orbital in the configuration space increases the number of virtual transitions that become available for the other orbitals. A similar effect occurs in the case of $\rho_{E 2}$, as it is seen in the inset of Fig. 7(d). In this case, the peak corresponds to the fluctuation between the configurations $A 1 E 1 E 2$ and $A 1 E 1$.

The change in the shape of $\rho_{E 1}$ in the 2 NEF approximation [Fig. 7(c)] is due to the elimination of the configurations with two holes in the E2 orbitals. The main peak in the spectral density is related to the fluctuation between the configurations $E 1 E 1 E 2$ and $E 1 E 2$. In the 4 NEF case, the contributions of the virtual fluctuations from $Q=E 1 E 2$ are given by $P^{\prime}=E 1 E 1 E 2$ and $P^{\prime}=E 1 E 2 E 2$, while in the 2 NEF case the contribution is only given by $P^{\prime}=E 1 E 1 E 2$. This difference introduced in the self-energy [Eq. (10)] is responsible for the change of the $\rho_{E 1}$ spectral density when going from the 4 NEF to the $2 \mathrm{NEF}$ approximation. For $\rho_{E 2}$ in Fig. 7(d), the main peak of the spectral density is related to the fluctuation between the configurations $E 1 E 1 E 2$ and $E 1 E 1$. These configurations do not present virtual fluctuations to states with two holes in $E 2$, so that the selfenergy corresponding to this transition is the same in both, $4 \mathrm{NEF}$ and $2 \mathrm{NEF}$ calculations. Notice that no change in the main peaks is observed when the space is restricted from 11 to 4 NEF. In this case, the virtual fluctuations that are neglected are the ones having $A 1$ as the active orbital, involving $\Gamma_{A 1} \approx$ $0 \mathrm{eV}$. Since $\Gamma_{A 1}$ is negligible compared with $\Gamma_{E 1}$ and $\Gamma_{E 2}$, the elimination of these transitions does not affect the main resonancelike peaks.

\section{Temperature behavior}

The spectral densities per symmetry group $\rho_{D}$, calculated from Eq. (20) at the reference values of the energy levels, are presented in Fig. 8 for different temperatures. Notice that at the extremely high temperatures included in the calculations, it is expected the adatom desorption, the formation of defects and the graphene decomposition [62,63]. However, these theoretical conditions are useful to test the behavior of the model. The resonancelike peaks that appear in the spectral densities are identified with the fluctuations of Table III. The total spectral density, obtained as the sum of the five orbital spectral densities, is presented in the same figure. The occurrence probabilities and hole occupations are also shown as a function of the temperature.
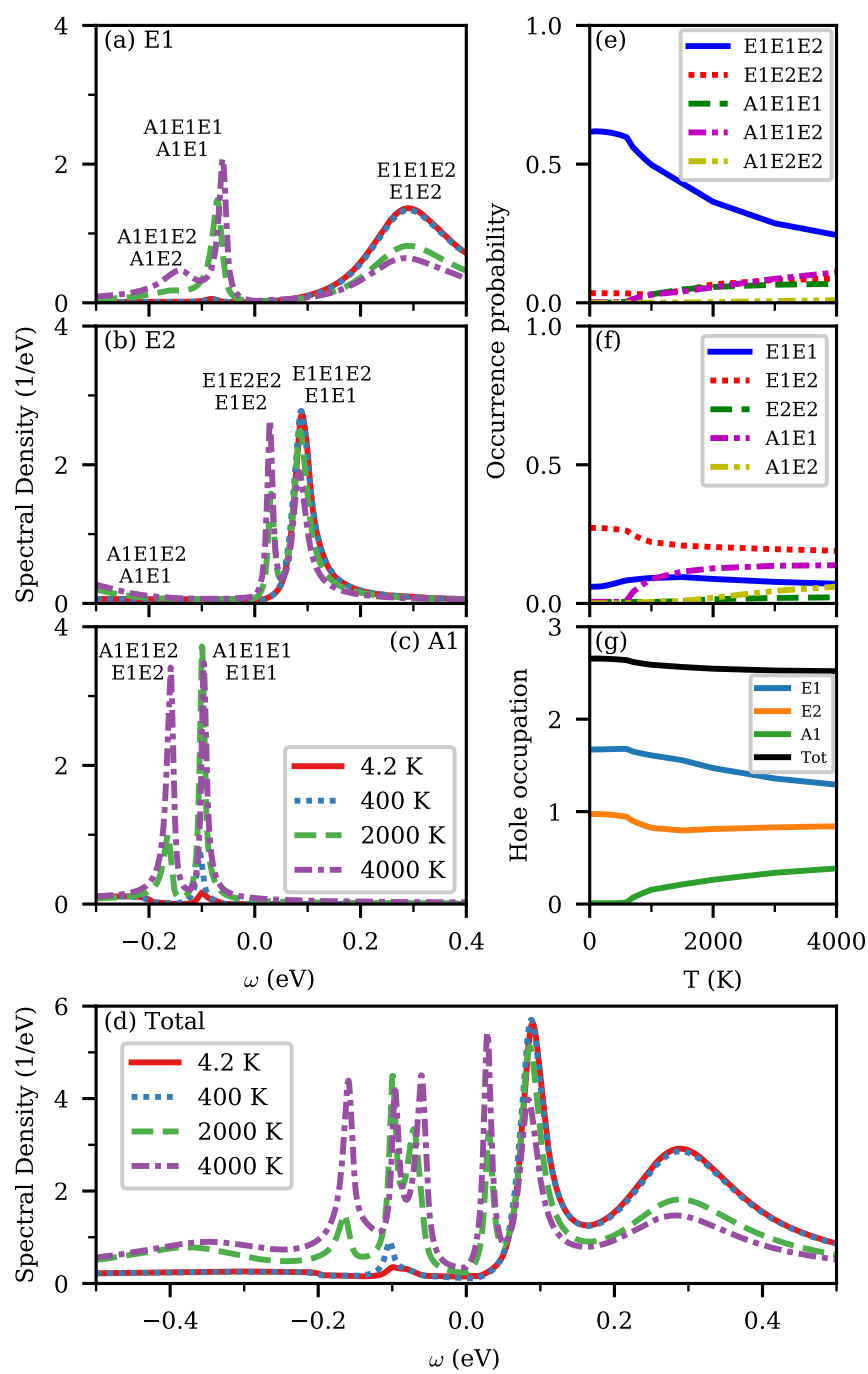

FIG. 8. [(a)-(c)] Spectral densities at several temperatures, for the three symmetry groups and (d) total spectral density. [(e) and (f)] Occurrence probabilities for the configurations with three and two holes as a function of the temperature. (g) Variation of the hole occupation as a function of the temperature.

In Figs. 8(a)-8(c), new peaks appear in the spectral densities as the temperature is increased and the occupation of the lower probability configurations becomes significant. In the case of $\rho_{E 1}$ [panel (a)], it is observed an enhancement of the small peak corresponding to the fluctuation between $A 1 E 1 E 1$ and $A 1 E 1$, discussed in Sec. V A. In addition, a fluctuation involving the configurations $A 1 E 1 E 2$ and $A 1 E 2$ becomes possible at high temperature. An analogous behavior is observed for $\rho_{E 2}$ and $\rho_{A 1}$ in panels (b) and (c). In Fig. 8(e), we observe that the occurrence probability of the three holes configuration $A 1 E 1 E 2$ increases from a negligible value at $4.2 \mathrm{~K}\left(\approx 10^{-3}\right)$ to 0.11 at $4000 \mathrm{~K}$. Since this configuration includes the three symmetry groups, its contribution can be observed in the three spectral densities [peaks labeled with $A 1 E 1 E 2$ in panels (a) to (c)]. The probability of $A 1 E 1 E 1$ also increases in a similar magnitude, and it is observed in the enhancement of the corresponding peaks in $\rho_{E 1}$ and $\rho_{A 1}$. In a similar way, the increase of the occurrence probability 
of the two holes configurations [Fig. 8(f)] is related to the enhancement of the corresponding peaks. This is the case of the $A 1 E 1$ configuration, which contributes to $\rho_{E 1}$ and $\rho_{E 2}$. The remaining fluctuations, such as between $A 1 E 2 E 2$ and $E 2 E 2$, are related to configurations with a very low occurrence probability even at high temperature, and are not observed in the spectral densities.

It is clear from Figs. 8(e) and 8(f) that the temperature increase produces a redistribution of the probabilities of the different configurations. The $E 1 E 1 E 2$ occupation, which at $4.2 \mathrm{~K}$ is 0.61 , decreases to 0.24 at $4000 \mathrm{~K}$ and becomes comparable to the occupation of practically the whole rest of three holes configurations $(\approx 0.1)$. The only exception is $A 1 E 2 E 2$, the configuration without holes in $E 1$, which is the least favorable with an occupation of $\approx 0.01$. Similarly, at high temperature the probabilities of the two holes configurations in Fig. 8(f) become comparable. As a result of the redistribution of probabilities, the temperature increase produces a slight decrease of the total hole occupation, from 2.65 to 2.52 [panel (g)].

The results presented in Fig. 8 show a good qualitative agreement with those of Ref. [8] (Fig. 4 of this reference), obtained using the GGA+OCA method. Our approach allows us to identify the development of the many-body structures in the spectral density with fluctuations between configurations which become accessible as the temperature is increased.

\section{CO-GRAPHENE SYSTEM OUT-OF-EQUILIBRIUM}

\section{A. Theoretical considerations and parameter calculation}

The Green-Keldysh functions (6) and (7) are appropriate for solving quantum systems out-of-equilibrium. The current $I$ between the graphene surface $(s)$ and a tip $(t)$ through the Co atom is computed from the time derivative of the average occupation number $\left\langle n_{v \mathbf{k} \sigma}\right\rangle$ in one of the leads $v=s, t$. The current from the lead $v$ to the other is given by [64]

$$
I_{\nu}=-\frac{2 e}{\hbar} \sum_{\substack{\mathbf{k}, \sigma, M \\ p, q \in p}} \operatorname{Im}\left\{V_{\nu \mathbf{k} M \sigma}^{p q *}\left\langle\mid \frac{3}{2}, M\right\rangle_{p}\left\langle 1, M-\left.\sigma\right|_{q} c_{\nu \mathbf{k} \sigma}\right\rangle\right\}
$$

This expression can be written as

$$
\begin{aligned}
I_{\nu}= & \frac{4 e}{h} \sum_{p, q \in p} \int_{-\infty}^{\infty} d \epsilon\left(\Lambda_{v d(p, q)}^{0}(\epsilon) \operatorname{Re} F_{p q}(\epsilon)\right. \\
& \left.+\Gamma_{v d(p, q)}^{0}(\epsilon) \operatorname{Im} K_{v p q}(\epsilon)\right),
\end{aligned}
$$

where $\quad K_{v p q}(\epsilon)=F_{p q}(\epsilon)-2\left(2 f_{v<}(\epsilon)-1\right) G_{p q}(\epsilon) \quad$ [see Eq. (A14) in Appendix A]. The Fermi function of each lead $f_{v<}$ is defined using the respective chemical potential $\mu_{\nu}$. In equilibrium the fluctuation-dissipation relation $F_{p q}(\epsilon)=2 i\left(2 f_{<}(\epsilon)-1\right) \operatorname{Im} G_{p q}(\epsilon)$ is satisfied and the current becomes zero.

We modeled the tip-atom-surface system by considering the atom-surface and the atom-tip interactions independently, which is an adequate approximation for the electronic transport in the tunneling regime [65]. For the calculation of the atom-tip system we followed a procedure similar to the one described in Sec. III for the computation of the Co-graphene Hamiltonian parameters. First, we performed a DFT compu-
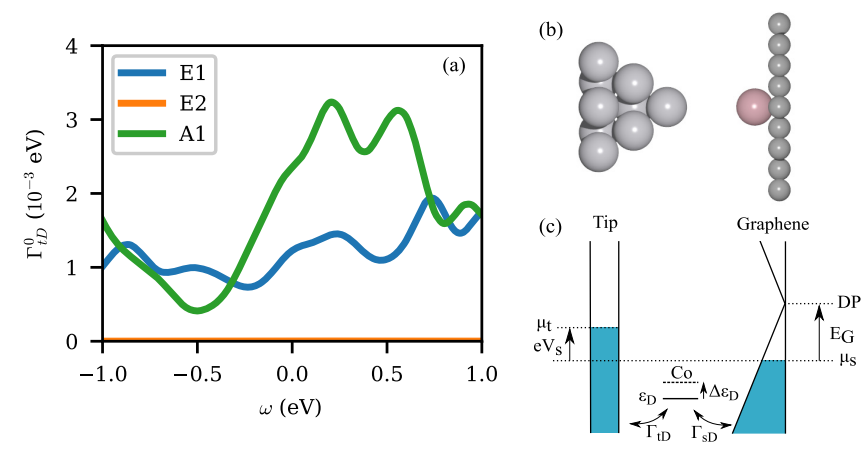

FIG. 9. (a) Co-tip noninteracting width $\Gamma_{t D}^{0}$ per symmetry group. (b) Geometry of the system. (c) Scheme indicating the position of the graphene Dirac point (DP) relative to the chemical potential of the surface $\mu_{s}$, the Co energy levels $\epsilon_{D}$ and their shift $\Delta \epsilon_{D}$, the position of the tip chemical potential $\mu_{t}$ when a bias voltage $V_{s}$ is applied and the interaction between the Co atom and each lead represented by $\Gamma_{s D}^{0}$ and $\Gamma_{t D}^{0}$.

tation of an isolated Pt tip. We modeled six layers of a $4 \times 4$ supercell of $\operatorname{Pt}(111)$, with three additional layers forming a pyramid, which is shown in Fig. 9(b). The three top layers were kept fixed at their bulk equilibrium positions and the rest of the atoms were allowed to relax. Once calculated the density matrix of the isolated Pt tip, the dimeric coupling between $\mathrm{Co}$ and $\mathrm{Pt}$ atoms were calculated by using the bondpair model described in Sec. IV A. Finally, the noninteracting self-energy (Eq. (12)) corresponding to the tip located on top of the Co atom was computed. The widths $\Gamma_{t D}^{0}$ due to the Co-tip interaction are shown for each symmetry group in Fig. 9(a), by considering a Co-tip distance of $5 \AA$.

Because of their symmetry, each Co orbital couples differently with the tip on top of the atom, being the $A 1$ orbital the most favorable. The $E 1$ orbitals, $d_{x z}$ and $d_{y z}$, are spatially located out of the surface plane and also couple with the tip. On the contrary, the $E 2$ orbitals are located on the surface plane and their couplings are negligible. On the other hand, the coupling of the $A 1$ orbital with the graphene is negligible near the Fermi level $\left[\Gamma_{S A 1}^{0}(0) \approx 0 \mathrm{eV}\right]$, so that this orbital does not provide a direct conduction channel [8]. Then, only the $E 1$ orbitals couple to both, the graphene and the tip, and are expected to provide the conduction channels registered by STM. This can be better understood using a simplified version of Eq. (29), where current conservation per channel and a near-equilibrium situation are assumed to calculate the current from $t$ to $s[39,66,67]$ :

$$
I=\frac{8 e}{h} \sum_{p, q \in p} \int_{-\infty}^{\infty} d \epsilon \Gamma_{d(p, q)}^{\mathrm{eff}}(\epsilon)\left(f_{t<}(\epsilon)-f_{s<}(\epsilon)\right) \operatorname{Im} G_{p q}(\epsilon) .
$$

The effective width introduced in Eq. (30) is given by $\Gamma_{d_{i}}^{\mathrm{eff}}=2 \Gamma_{t d_{i}}^{0} \Gamma_{s d_{i}}^{0} /\left(\Gamma_{t d_{i}}^{0}+\Gamma_{s d_{i}}^{0}\right)$ [39]. For the $E 1$ and $E 2$ orbitals, $\Gamma_{t d_{i}}^{0}<<\Gamma_{s d_{i}}^{0}$ and therefore $\Gamma_{d_{i}}^{\mathrm{eff}} \approx \Gamma_{t d_{i}}^{0}$. Then, the E2 orbitals do not provide an effective current channel due to the negligible interaction of these orbitals with the tip. The same conclusion applies for the $A 1$ orbital, in this case due to the negligible interaction with the surface, $\Gamma_{A 1}^{\text {eff }} \approx \Gamma_{s d_{i}}^{0}$. 
In summary, only the $E 1$ channels contribute significantly to the electronic transport through the Co atom.

\section{B. Analysis of the differential conductance}

The STM spectra of Co atoms deposited on back-gated graphene grown on $\mathrm{SiO}_{2}$ have been measured for different back-gate voltages $V_{G}$ [18]. Among the several features found in the differential conductance spectra shown in Ref. [18], we will focus on the resonance peaks (labeled with $\mathrm{A}, \mathrm{B}, \mathrm{C}$, and D) and the related S peak of Fig. 2 of the cited work, associated with the electronic structure of the Co impurity.

The gate potential $V_{G}$ applied in the experiment shifts the chemical potential $\mu_{s}$ of graphene [18]. The approaches used to model the $\mu$ shift include the calculation of the system for different values of electron doping $[13,14]$ and the direct variation of $\mu_{s}$ [8], which is used in this work. It is worth noting that the $V_{G}$-induced shift of $\mu_{s}$ is not the only effect to take into account. As pointed out in Ref. [18], the work function difference between the surface and the tip and the application of the bias voltage $V_{s}$ also induces a $\mu$ shift, that varies with the value of $V_{s}$. Consistently with Ref. [18], we define the bias potential $V_{s}$ as the voltage of the surface with respect to the tip, so that the chemical potential of the tip corresponds to $\mu_{t}=e V_{s}$ ( $\mu_{s}$ is chosen as zero for all the calculations). In addition, when the tip is right above the Co adatom, the atom states are shifted in energy [18]. Also, when $V_{G}$ varies, the change in the doping induces an additional shifting in the Co levels.

We consider independent variations of $\mu_{s}$ and $\epsilon_{D}$, in order to facilitate the analysis and identify the influence of each parameter in the calculated spectra. We use as reference the Dirac point, and define $E_{G}$ as the shift of the Dirac point with respect to the Fermi level of the graphene due to the gate potential. For a negative gate potential $\left(V_{G}<0\right)$, the corresponding shift is towards positive values $\left(E_{G}>0\right)$ and the Dirac point is at higher energies with respect to $\mu_{s}=0 \mathrm{eV}$ [see Fig. 9(c)]. The magnitude of $E_{G}$ can be estimated from the shifting of the Dirac point reported in Ref. [18], where the maximum shift measured was $\approx 0.25 \mathrm{eV}$.

In addition, we have to consider the shift of the Co energy levels $\Delta \epsilon_{D}$. The shift due to the presence of the Pt tip can be estimated using the bond-pairs model, as it was done in Sec. IV B to obtain the energy levels. For the tip at $5 \AA$ from the Co adatom, the resulting value is $\Delta \epsilon_{D}=0.03 \mathrm{eV}$. The change in the graphene doping by the applied $V_{G}$ and the variation of $V_{s}$ also affect the adatom levels [18]. To analyze these effects we proposed a variation of $\Delta \epsilon_{D}$ between $-0.1 \mathrm{eV}$ and $0.1 \mathrm{eV}$. In this range of analyzed values of $E_{G}$ and $\Delta \epsilon_{D}$, no Kondo-like structures are expected (see Sec. V A).

In Fig. 10, we present the differential conductance $d I / d V_{s}$ as a function of $V_{s}$, computed from Eq. (29) for several values of $E_{G}$ and $\Delta \epsilon_{D}$. The conditions $E_{G}=0 \mathrm{eV}$ and $\Delta \epsilon_{D}=$ $0 \mathrm{eV}$ (bottom panel of Fig. 10, full line curve) are the same as in the equilibrium calculations at the reference energy levels of Sec. V. We identify the peak that appears in the spectrum at $V_{s}=-0.08 \mathrm{eV}$ with the fluctuation between the configurations $A 1 E 1 E 1$ and $A 1 E 1$ [see inset of Fig. 5(a)].

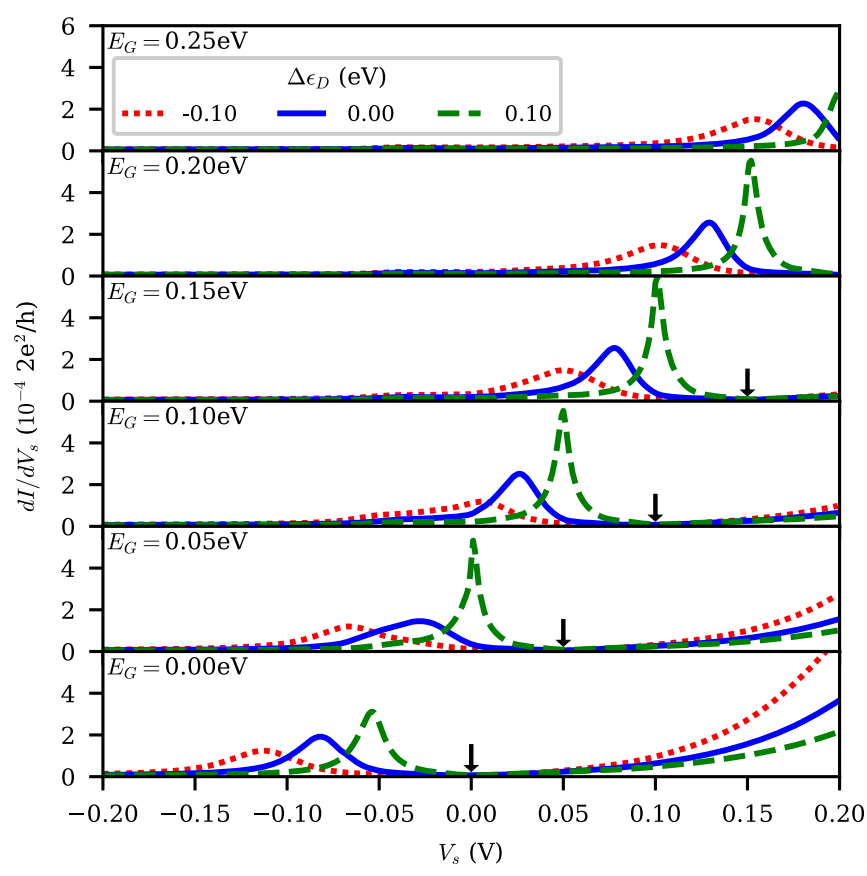

FIG. 10. Differential conductance for several $E_{G}$ shifts. The Dirac point position with respect to $\mu_{s}=0 \mathrm{eV}$ corresponds to $E_{G}$ and is indicated with an arrow. The calculations are made for different rigid shifts of the Co energy levels $\Delta \epsilon_{D}$.

The low occurrence probabilities of these configurations lead to the small intensity of the resonance. Notice that the peak width $(0.03 \mathrm{~V}$ at half maximum) resembles the width of the resonance features observed in Ref. [18] (peaks labeled with $\mathrm{A}, \mathrm{B}, \mathrm{C}, \mathrm{D})$. The increase in the conductance at high $V_{s}$ values is due to the peak associated with the fluctuation between the $E 1 E 1 E 2$ and $E 1 E 2$ configurations [see Fig. 5(a)].

We see that the peak related to the configurations $A 1 E 1 E 1$ and $A 1 E 1$, located at $V_{s}=-0.08 \mathrm{~V}$ for $E_{G}=0 \mathrm{eV}$, is shifted towards higher values of $V_{s}$ when $E_{G}$ is increased. Its displacement follows that of the Dirac point, which is located at $E_{G}$ by definition. By considering the variation of $E_{G}$ from 0.00 to $0.25 \mathrm{eV}$, the position of the peak observed in our calculation is in good agreement with the peak labeled with A in Ref. [18]. However, we should point out that the variation of $V_{G}$ in the experiment is not directly related to this $E_{G}$ variation. Instead, the measurement starts at a value of $E_{G}>0 \mathrm{eV}$, due to the doping of the graphene grown on the $\mathrm{SiO}_{2}$ substrate and the presence of the $\mathrm{Pt}$ tip. On the other hand, and as it was stated above, there is an additional contribution to the energy shift $E_{G}$ that depends on the bias voltage $V_{s}$ [18]. For $V_{s}<0 \mathrm{~V}$, the additional band-shift due to $V_{s}$ leaves the Dirac point closer to $\mu_{s}$, as if $E_{G}$ were smaller. The shifting is in the opposite direction when $V_{s}>0 \mathrm{~V}$ : the Dirac point is further from $\mu_{s}$, as if $E_{G}$ were larger. As a result, the features observed at a given $E_{G}$ will correspond to the spectra calculated at a smaller $E_{G}$ for $V_{s}<0 \mathrm{~V}$ and at a larger $E_{G}$ for $V_{s}>0 \mathrm{~V}$. The magnitude of this shift is $\approx 0.05 \mathrm{eV}$ for the range of $V_{s}$ explored in the experiment [18]. Then, a measure at, for example, $E_{G}=0.05 \mathrm{eV}$ will be more similar to our result with $E_{G}=0.10 \mathrm{eV}$ for $V_{s}>0 \mathrm{~V}$ and with $E_{G}=0.00 \mathrm{eV}$ for $V_{s}<0 \mathrm{~V}$. Taking into account this effect, the peak observed 
in our calculation is in qualitative agreement with the one observed in the measurement. Nevertheless, we have to say that the uncertainty in the experimental positions of the peaks together with the approximated determination of the chemical potential and the level shifts in the theoretical model, make difficult a direct comparison.

The resonance observed in our calculation is produced by fluctuations involving the low-probability configurations $A 1 E 1 E 1$ and $A 1 E 1$, suggesting that further features could appear if more configurations were considered. This assertion can be tested by expanding the space of configurations used in this work, which was restricted to the states with two and three holes. It would be of particular interest to include in the selected space the configurations with a single hole, allowing transitions to states with $S=1 / 2$, which are suggested for this system in other theoretical calculations [3,12,15-17].

We discuss now the effect of the energy shift $\Delta \epsilon_{D}$ in Fig. 10, in relation to the $\mathrm{S}$ peak observed in the experiment [18]. The $\mathrm{S}$ peak is related to the ionization of the Co atom when $V_{s}$ is great enough to cause the resonance feature to cross the Fermi level [18]. The $\mathrm{S}$ peak is observed at $V_{s}>0 \mathrm{~V}$ when the resonance is below $0 \mathrm{eV}$ and vice versa. Take as example the spectrum for $E_{G}=0.05 \mathrm{eV}$ in Fig. 10, expected for a $E_{G}=$ $0.00 \mathrm{eV}$ in the measurement made for $V_{s}>0 \mathrm{~V}$. At sufficiently high $V_{s}$, the Co energy levels are shifted closer to the Dirac point (curve corresponding to $\Delta \epsilon_{D}=0.10 \mathrm{eV}$ ), where the interaction width is lower and consequently the resonance is sharper. Then, the bias potential applied, $V_{s}>0 \mathrm{~V}$, shifts and sharpen the resonance, which is seen as the narrow $S$ peak in the spectra when it crosses the Fermi level. On the other hand, when the measurement is made at a higher $E_{G}$ and for $V_{s}<0 \mathrm{~V}$, the resonance now located above $0 \mathrm{eV}$ will be shifted towards lower energies until it crosses the Fermi level. Considering the value $E_{G}=0.15 \mathrm{eV}$ in Fig. 10, the peak located at $V_{s}=0.08 \mathrm{~V}$ when $\Delta \epsilon_{D}=0 \mathrm{eV}$ will be shifted towards lower values and broadened when the measure is done at $V_{s}<0 \mathrm{~V}$. Then, the related $\mathrm{S}$ peak, measured at $V_{s}<0 \mathrm{~V}$ when the resonance crosses the Fermi level, will be wider than the original peak, like the spectrum for $\Delta \epsilon_{D}=-0.10 \mathrm{eV}$ in our calculation.

\section{CONCLUSIONS}

We analyzed the multiorbital electronic correlation in a Co atom adsorbed on graphene. From DFT calculations, we obtained the adatom equilibrium position and orbital occupations, which suggested that the more probable electronic configurations of the Co adatom are those with total spin $S=3 / 2$ and $S=1$. Then, we described the interacting system by using the Anderson Hamiltonian in its ionic form, obtained by projecting it in the selected space of atomic configurations. The Green-Keldysh functions used to obtained the physical quantities were solved using the Equation of Motion method. This approach allowed a precise identification of the configurations and the fluctuations between them, which determine the electronic structure of the system.

The Hamiltonian parameters were obtained by using the bond-pair model, which allowed us to analyze the coupling of the Co orbitals with the different graphene bands without supercell effects and to determine the one particle energy levels involved in the adsorbate spin and charge fluctuations. It was observed the preferential coupling of the $E 1$ orbitals to the graphene $\pi$ band and of the $E 2$ orbitals to the $\pi^{*}$ band. In spite of the shared symmetry, the orbitals of each symmetry group couples with different regions of the first Brillouin zone, in correspondence with the geometry of the orbitals. Naturally, the different couplings lead to a noninteracting hybridization function only dependent on the orbital symmetry.

The calculations in the equilibrium situation were in agreement with previous findings and allowed to elucidate some aspects of the Co on graphene system. At the calculated reference energy level positions, the system is in a mixed valence regime, with a preferential occupation of the $S=$ $3 / 2$ configuration $E 1 E 1 E 2$. When lowering the energy levels, there is a transition to a ground state with a larger weight of the $S=1$ configurations, in which the two holes are preferentially located in the $E 1$ orbitals. In the opposite case, when the ground state is mainly defined by the $S=3 / 2$ configurations, the predicted Kondo resonance only appears in a doped graphene with an appreciable DOS at the Fermi level.

The calculated spectral densities showed several resonancelike peaks, related to the fluctuations between the different configurations. This many-body signature in the system is a direct expression of the configurations with higher probability. It was observed a small peak in the spectral density of the $E 1$ orbitals, related to virtual fluctuations between the $A 1 E 1 E 1$ and $A 1 E 1$ configurations, both with a low occurrence probability. This peak is absent when the configurations with holes in the $A 1$ orbital are not considered, showing the importance of selecting an adequate space for projecting the Anderson Hamiltonian.

The suppression of the main peak in the $E 1$ spectral density when lowering the energy levels, previously attributed to a variation in the interaction width, was found to be instead a phenomenon inherent to the multiorbital characteristic of the system: the $E 1$ orbitals are completely occupied, and the fluctuations can only occur in the other orbitals. The marked temperature dependence of the spectral densities is another indication of electronic correlation effects. It was identified a direct relation of the enhancement with the temperature of the different peaks in the spectral densities with the increase in the probability of the configurations involved in the fluctuations.

The differential conductance spectra given by a bias potential applied to a $\mathrm{Pt}$ tip on top of the Co adatom were calculated using the Keldysh formalism. Only the $E 1$ orbitals couple with both, the surface and the tip, and therefore can contribute to the current measured in a STM experiment. The peak related to fluctuations involving the low-probability configurations $A 1 E 1 E 1$ and $A 1 E 1$ was related to resonancelike features observed in the measured conductance spectra.

\section{ACKNOWLEDGMENTS}

This work was supported by Consejo Nacional de Investigaciones Científicas y Técnicas (CONICET) through PIP grants, Universidad Nacional del Litoral (UNL) through CAI+D grants, and the German Academic Exchange Service (DAAD). 


\section{APPENDIX A: GREEN FUNCTIONS CALCULATION}

The required Green functions $G_{p q}$ and $F_{p q}$ of Eqs. (6) and (7) are calculated using the following expressions:

$$
\begin{gathered}
\left(\omega-\epsilon_{d(p, q)}-\Sigma_{p q}(\omega)\right) G_{p q}(\omega)=O_{p q}+\Xi_{p q}(\omega), \\
\left(\omega-\epsilon_{d(p, q)}-\Sigma_{p q}^{*}(\omega)\right) F_{p q}(\omega)=\Omega_{p q}(\omega) G_{p q}(\omega)+\Upsilon_{p q}(\omega) .
\end{gathered}
$$

The occupation term, $O_{p q}=\left\langle\frac{3}{2}\right\rangle_{p}+\langle 1\rangle_{q}$, is calculated from the occurrence probabilities, given by

$$
\begin{aligned}
\left\langle\frac{3}{2}\right\rangle_{p} & =\frac{1}{\pi} \int_{-\infty}^{\infty} d \omega \operatorname{Im} G_{p q}^{<}(\omega), \\
\langle 1\rangle_{q} & =\frac{1}{\pi} \int_{-\infty}^{\infty} d \omega \operatorname{Im} G_{p q}^{>}(\omega) .
\end{aligned}
$$

Here, $\operatorname{Im} G_{p q}^{\lessgtr}(\omega)=\frac{1}{4} \operatorname{Im}\left\{F_{p q}(\omega) \pm 2 G_{p q}(\omega)\right\}$ for the out-of-equilibrium situation. In equilibrium,

$$
F_{p q}(\omega)=2 i\left(2 f_{<}(\omega)-1\right) \operatorname{Im} G_{p q}(\omega),
$$

and $\operatorname{Im} G_{p q}^{\lessgtr}(\omega)=f_{\lessgtr}(\omega) \operatorname{Im} G_{p q}(\omega)$. The terms $\Sigma_{p q}$ and $\Omega_{p q}$ can be expressed as

$$
X_{p q}(\omega)=\sum_{\nu} \sum_{q^{\prime} \in p} X_{v d\left(p^{\prime} q\right)}^{>}\left(\omega+\epsilon_{d\left(p^{\prime} q\right)}-\epsilon_{d(p q)}\right)+\frac{4}{3} \sum_{\nu} \sum_{p^{\prime} \ni q} X_{\nu d\left(p^{\prime} q\right)}^{<}\left(\omega+\epsilon_{d\left(p^{\prime} q\right)}-\epsilon_{d(p q)}\right),
$$

where $X$ should be replaced by $\Sigma$ and $\Omega$, respectively. The index $v=t, s$ denotes the surface and tip sides in the out-ofequilibrium case, and reduces to only the surface in equilibrium. The self-energy $\Sigma_{\nu d_{i}}^{<[>]}$and its out-of-equilibrium counterpart $\Omega_{\nu d}^{<[>]}$are given by

$$
\begin{gathered}
\Sigma_{\nu d_{i}}^{<[>]}(\tilde{\omega})=\int_{-\infty}^{\infty} d \epsilon \frac{f_{\nu<[>]}(\epsilon) \Gamma_{\nu d_{i}}^{0}(\epsilon)}{\tilde{\omega}-\epsilon-i \eta}, \\
\Omega_{\nu d_{i}}^{<[>]}(\tilde{\omega})=2 i\left(2 f_{\nu<}(\tilde{\omega})-1\right) f_{\nu<[>]}(\tilde{\omega}) \Gamma_{\nu d}^{0}(\tilde{\omega}),
\end{gathered}
$$

where the width $\Gamma_{\nu d}^{0}(\tilde{\omega})$ is the imaginary part of the noninteracting self-energy of each side,

$$
\Sigma_{\nu d_{i}}^{0}(\omega)=\sum_{\mathbf{k}} \frac{\left|V_{\nu \mathbf{k} d_{i}}\right|^{2}}{\omega-\epsilon_{\nu \mathbf{k}}-i \eta}
$$

In the derivation of Eq. (A6), the degeneracy in $\sigma$ and $M$ allows us to do the following sum:

$$
\begin{gathered}
\sum_{\sigma^{\prime}}\left|V_{\nu \mathbf{k} M^{\prime} \sigma^{\prime}}^{p q}\right|^{2}=V_{\nu \mathbf{k} d(p, q),} \\
\sum_{\sigma^{\prime}} \mid V_{\nu \mathbf{k}\left(M^{\prime}+\sigma^{\prime}\right) \sigma^{\prime}}^{p q}=\frac{4}{3} V_{\nu \mathbf{k} d(p, q)},
\end{gathered}
$$

where $V_{\nu \mathbf{k} M{ }^{\prime} \sigma^{\prime}}^{p q}$ is given by Eq. (4). The result is independent of $M^{\prime}, \sigma^{\prime}$ and introduces the factors 1 and 4/3 of Eq. (A6).

In the derivation of the equations of motion, crossed atom-band terms with the form

$$
C_{\nu \mathbf{k} \sigma}^{p q M \sigma}=\left\langle\mid \frac{3}{2}, M\right\rangle_{p}\left\langle 1, M-\left.\sigma\right|_{q} c_{\nu \mathbf{k} \sigma}\right\rangle
$$

are obtained [38]. In equilibrium these terms are calculated as follows:

$$
C_{\nu \mathbf{k} \sigma}^{p q M \sigma}=\frac{1}{\pi} V_{\nu \mathbf{k} M \sigma}^{p q} \operatorname{Im}\left\{\int_{-\infty}^{\infty} d \omega^{\prime} f_{\nu<}\left(\omega^{\prime}\right) \frac{G_{p q}^{M \sigma}\left(\omega^{\prime}\right)}{\omega^{\prime}-\epsilon_{\mathbf{k}}-i \eta}\right\}
$$

while for the out-of-equilibrium situation the expression is the following:

$$
C_{\nu \mathbf{k} \sigma}^{p q M \sigma}=-i \frac{1}{4 \pi} V_{\nu \mathbf{k} M \sigma}^{p q} \int_{-\infty}^{\infty} d \omega^{\prime} \frac{\left(F_{p q}^{M \sigma}\left(\omega^{\prime}\right)-\left(2 f_{\nu<}\left(\epsilon_{\mathbf{k}}\right)-1\right) G_{p q}^{M \sigma}\left(\omega^{\prime}\right)\right)}{\omega^{\prime}-\epsilon_{\mathbf{k}}+i \eta} .
$$

Again using degeneracy, we can express the contribution of these crossed atom-band terms, given by $\Xi_{p q}$ and $\Upsilon_{p q}$ in Eqs. (A1) and (A2), as

$$
Y_{p q}(\omega)=\sum_{\nu} \sum_{q^{\prime} \in p} Y_{\nu p^{\prime} q}^{C}\left(\omega+\epsilon_{d\left(p^{\prime} q\right)}-\epsilon_{d(p q)}\right)-\frac{4}{3} \sum_{\nu} \sum_{p^{\prime} \ni q} Y_{\nu p^{\prime} q}^{C}\left(\omega+\epsilon_{d\left(p^{\prime} q\right)}-\epsilon_{d(p q)}\right)
$$


where $Y$ should be replaced by $\Xi$ and $\Upsilon$, respectively. The terms $\Xi_{v p^{\prime} q^{\prime}}^{C}$ and $\Upsilon_{v p^{\prime} q^{\prime}}^{C}$ are given by

$$
\begin{gathered}
\Xi_{v p^{\prime} q^{\prime}}^{C}(\tilde{\omega})=\Xi_{v p^{\prime} q^{\prime}}^{A}(\tilde{\omega})+i \Xi_{v p^{\prime} q^{\prime}}^{B}(\tilde{\omega}), \\
\Upsilon_{v p^{\prime} q^{\prime}}^{C}(\tilde{\omega})=2 i\left(2 f_{v<}(\tilde{\omega})-1\right)\left(\operatorname{Im} \Xi_{v p^{\prime} q^{\prime}}^{A}+i \operatorname{Im} \Xi_{v p^{\prime} q^{\prime}}^{B}\right),
\end{gathered}
$$

with

$$
\begin{aligned}
\Xi_{v p^{\prime} q^{\prime}}^{A}(\tilde{\omega})= & +\frac{1}{\pi} \int_{-\infty}^{\infty} d \omega^{\prime} \frac{f_{v<}\left(\omega^{\prime}\right)}{\omega^{\prime}-\tilde{\omega}+i \eta}\left(\Sigma_{v d\left(p^{\prime} q^{\prime}\right)}^{0}(\tilde{\omega}) \operatorname{Im} G_{p^{\prime} q^{\prime}}\left(\omega^{\prime}\right)-\operatorname{Im}\left\{\Sigma_{v d\left(p^{\prime} q^{\prime}\right)}^{0}\left(\omega^{\prime}\right) G_{p^{\prime} q^{\prime}}\left(\omega^{\prime}\right)\right\}\right) \\
& +\frac{1}{4 \pi} \int_{-\infty}^{\infty} d \omega^{\prime} \frac{1}{\omega^{\prime}-\tilde{\omega}+i \eta} \Gamma_{v d\left(p^{\prime} q^{\prime}\right)}^{0}\left(\omega^{\prime}\right) \operatorname{Re} F_{p^{\prime} q^{\prime}}\left(\omega^{\prime}\right) \\
& +\frac{1}{4 \pi} \int_{-\infty}^{\infty} d \omega^{\prime} \frac{1}{\omega^{\prime}-\tilde{\omega}+i \eta}\left(\Sigma_{v d\left(p^{\prime} q^{\prime}\right)}^{0}(\tilde{\omega})-\Lambda_{v d\left(p^{\prime} q^{\prime}\right)}^{0}\left(\omega^{\prime}\right)\right)\left(\operatorname{Im} F_{p^{\prime} q^{\prime}}\left(\omega^{\prime}\right)-2\left(2 f_{v}^{<}\left(\omega^{\prime}\right)-1\right) \operatorname{Im} G_{p^{\prime} q^{\prime}}\left(\omega^{\prime}\right)\right), \\
\Xi_{v p^{\prime} q^{\prime}}^{B}(\tilde{\omega})= & \frac{1}{4 \pi} \int_{-\infty}^{\infty} d \omega^{\prime} \frac{1}{\omega^{\prime}-\tilde{\omega}+i \eta}\left(\Lambda_{v d\left(p^{\prime} q^{\prime}\right)}^{0}\left(\omega^{\prime}\right)-\Sigma_{v d\left(p^{\prime} q^{\prime}\right)}^{0}(\tilde{\omega})\right) \operatorname{Re} F_{p^{\prime} q^{\prime}}\left(\omega^{\prime}\right) \\
& +\frac{1}{4 \pi} \int_{-\infty}^{\infty} d \omega^{\prime} \frac{1}{\omega^{\prime}-\tilde{\omega}+i \eta} \Gamma_{v d\left(p^{\prime} q^{\prime}\right)}^{0}\left(\omega^{\prime}\right)\left(\operatorname{Im} F_{p^{\prime} q^{\prime}}\left(\omega^{\prime}\right)-2\left(2 f_{v}^{<}\left(\omega^{\prime}\right)-1\right) \operatorname{Im} G_{p^{\prime} q^{\prime}}\left(\omega^{\prime}\right)\right) .
\end{aligned}
$$

In equilibrium the term $\Xi_{v p^{\prime} q^{\prime}}^{A}$ reduces to only the first line, while $\Xi_{v p^{\prime} q^{\prime}}^{B}=0$.

\section{APPENDIX B: TOTAL ENERGY CALCULATION}

After the many body Hamiltonian that describes the atom-surface interacting system is written as a superposition of bond pairs, all the parameters except the hopping terms are expanded up to a second order in the atomic overlap $S_{d_{i} \alpha s}$ (recall that $\alpha$ labels the orbitals of the $s$ atom of the surface) [41,68]. The overlap expansion was successfully used with overlap values as large as 0.7 [41]. The total energies are finally given by the expression

$$
E_{S, p[q]}=\sum_{d_{i}, \sigma}\left\langle n_{d_{i} \sigma}\right\rangle\left(\epsilon_{d_{i} \sigma, p[q]}^{\mathrm{HFC}}-\sum_{s} V_{Z_{s} d_{i}}+\sum_{\alpha, s}\left(J_{d_{i} \alpha s}^{0}\left\langle n_{\alpha s \bar{\sigma}}\right\rangle+J_{d_{i} \alpha s}^{\prime}\left\langle n_{\alpha s \sigma}\right\rangle\right)-\sum_{\alpha, s} S_{d_{i} \alpha s} V_{\alpha s d_{i}}+\frac{1}{4} \sum_{\alpha, s} S_{d_{i} \alpha s}^{2} \Delta E_{d_{i} \alpha s, p[q]}\right) .
$$

The short-range electron-nuclei interactions $\left(V_{Z_{s} d_{i}}\right)$ and the electron-electron direct $\left(J_{d_{i} \alpha s}\right)$ and exchange $\left(J_{d_{i} \alpha s}^{x}\right)$ interactions are included. We define $J_{d_{i} \alpha s}^{\prime}=J_{d_{i} \alpha s}-J_{d_{i} \alpha s}^{x}$. Both $J_{d_{i} \alpha s}$ and $J_{d_{i} \alpha s}^{\prime}$ are also calculated up to a second order in $S_{d_{i} \alpha s}$. The terms with superscript 0 , as $J_{d_{i} \alpha s}^{0}$, correspond to the zero order in $S_{d_{i} \alpha s}$. In the last term of Eq. (B1), $\Delta E_{d_{i} \alpha s, p[q]}$ is the difference between the energies of the adatom and the surface atoms.

The orbital occupation $\left\langle n_{d_{i} \sigma}\right\rangle$ corresponds to the occupation of the $d_{i}$ orbital of the atom in the $p[q]$ configuration. Similarly, $\left\langle n_{\alpha s \sigma}\right\rangle$ corresponds to the average occupation of the atomic orbitals of the surface atoms. In addition, $\epsilon_{d_{i} \sigma, p[q]}^{\mathrm{HFC}}$ is the Hartree-Fock orbital energy $\epsilon_{d_{i} \sigma, p[q]}^{\mathrm{HF}}$ corrected by double counting:

$$
\epsilon_{d_{i} \sigma, p[q]}^{\mathrm{HFC}}=\epsilon_{d_{i} \sigma, p[q]}^{\mathrm{HF}}-\frac{1}{2} U_{d_{i}}^{0}\left\langle n_{d_{i} \bar{\sigma}}\right\rangle+\frac{1}{2} \sum_{d_{j} \neq d_{i}}\left(J_{d_{i} d_{j}}^{0}\left\langle n_{d_{j} \bar{\sigma}}\right\rangle+J_{d_{i} d_{j}}^{\prime}\left\langle n_{d_{j} \sigma}\right\rangle\right),
$$

being $U_{d_{i}}^{0}$ the Coulomb interaction in the same orbital $d_{i}$. See Ref. [41] for details on the method.

[1] W. Han, R. K. Kawakami, M. Gmitra, and J. Fabian, Nat. Nanotechnol. 9, 794 (2014).

[2] B. Dlubak, M. B. Martin, C. Deranlot, B. Servet, S. Xavier, R. Mattana, M. Sprinkle, C. Berger, W. A. De Heer, F. Petroff, A. Anane, P. Seneor, and A. Fert, Nat. Phys. 8, 557 (2012).

[3] T. O. Wehling, A. I. Lichtenstein, and M. I. Katsnelson, Phys. Rev. B 84, 235110 (2011).

[4] O. V. Yazyev and L. Helm, Phys. Rev. B 75, 125408 (2007).

[5] J. Ren, H. Guo, J. Pan, Y. Y. Zhang, X. Wu, H. G. Luo, S. Du, S. T. Pantelides, and H. J. Gao, Nano Lett. 14, 4011 (2014).
[6] F. Donati, Q. Dubout, G. Autès, F. Patthey, F. Calleja, P. Gambardella, O. V. Yazyev, and H. Brune, Phys. Rev. Lett. 111, 236801 (2013).

[7] D. Pesin and A. H. MacDonald, Nat. Mater. 11, 409 (2012).

[8] D. Jacob and G. Kotliar, Phys. Rev. B 82, 085423 (2010).

[9] A. C. Hewson, The Kondo Problem to Heavy Fermions (Cambridge University Press, Cambridge, UK, 1993).

[10] J. C. Cuevas and E. Scheer, Molecular Electronics (World Scientific, Singapore, 2010).

[11] R. Mozara, M. Valentyuk, I. Krivenko, E. Sasioglu, J. Kolorenc, and A. I. Lichtenstein, Phys. Rev. B 97, 085133 (2018). 
[12] T. O. Wehling, A. V. Balatsky, M. I. Katsnelson, A. I. Lichtenstein, and A. Rosch, Phys. Rev. B 81, 115427 (2010).

[13] A. Saffarzadeh and G. Kirczenow, Phys. Rev. B 85, 245429 (2012).

[14] K. T. Chan, H. Lee, and M. L. Cohen, Phys. Rev. B 83, 035405 (2011).

[15] A. N. Rudenko, F. J. Keil, M. I. Katsnelson, and A. I. Lichtenstein, Phys. Rev. B 86, 075422 (2012).

[16] Y. Virgus, W. Purwanto, H. Krakauer, and S. Zhang, Phys. Rev. Lett. 113, 175502 (2014).

[17] Y. Virgus, W. Purwanto, H. Krakauer, and S. Zhang, Phys. Rev. B 86, 241406(R) (2012).

[18] V. W. Brar, R. Decker, H. M. Solowan, Y. Wang, L. Maserati, K. T. Chan, H. Lee, Ã. O. Girit, A. Zettl, S. G. Louie, M. L. Cohen, and M. F. Crommie, Nat. Phys. 7, 43 (2011).

[19] T. O. Wehling, H. P. Dahal, A. I. Lichtenstein, M. I. Katsnelson, H. C. Manoharan, and A. V. Balatsky, Phys. Rev. B 81, 085413 (2010).

[20] Y. Yagi, T. M. Briere, M. H. F. Sluiter, V. Kumar, A. A. Farajian, and Y. Kawazoe, Phys. Rev. B 69, 075414 (2004).

[21] L. Hu, X. Hu, X. Wu, C. Du, Y. Dai, and J. Deng, Phys. B: Condens. Matter 405, 3337 (2010).

[22] A. I. Liechtenstein, V. I. Anisimov, and J. Zaanen, Phys. Rev. B 52, R5467 (1995).

[23] P. W. Anderson, Phys. Rev. 124, 41 (1961).

[24] K. G. Wilson and J. Kogut, Phys. Rep. 12, 75 (1974).

[25] R. Bulla, T. A. Costi, and T. Pruschke, Rev. Mod. Phys. 80, 395 (2008)

[26] M. Caffarel and W. Krauth, Phys. Rev. Lett. 72, 1545 (1994).

[27] P. Coleman, Phys. Rev. B 29, 3035 (1984).

[28] K. Haule, S. Kirchner, J. Kroha, and P. Wölfle, Phys. Rev. B 64, 155111 (2001).

[29] D. Jacob, K. Haule, and G. Kotliar, Phys. Rev. Lett. 103, 016803 (2009)

[30] C. Lacroix, J. Phys. F: Met. Phys. 11, 2389 (2000).

[31] K. Kang and B. I. Min, Phys. Rev. B 52, 10689 (1995).

[32] E. C. Goldberg, F. Flores, and R. C. Monreal, Phys. Rev. B 71, 035112 (2005).

[33] Q. Feng, Y. Z. Zhang, and H. O. Jeschke, Phys. Rev. B 79, 235112 (2009).

[34] Q. Feng and P. M. Oppeneer, Phys. Rev. B 86, 035107 (2012).

[35] Y. Meir, N. S. Wingreen, and P. A. Lee, Phys. Rev. Lett. 70, 2601 (1993).

[36] L. L. Hirst, Adv. Phys. 27, 231 (1978).

[37] L. V. Keldysh, J. Exp. Theor. Phys. 20, 1018 (1965).

[38] M. A. Romero, F. Flores, and E. C. Goldberg, Phys. Rev. B 80, 235427 (2009).

[39] E. C. Goldberg and F. Flores, J. Phys.: Condens. Matter 25, 225001 (2013).

[40] E. C. Goldberg and F. Flores, Phys. Rev. B 96, 115439 (2017).

[41] P. G. Bolcatto, E. C. Goldberg, and M. C. G. Passeggi, Phys. Rev. B 58, 5007 (1998).
[42] G. Kotliar, S. Y. Savrasov, K. Haule, V. S. Oudovenko, O. Parcollet, and C. A. Marianetti, Rev. Mod. Phys. 78, 865 (2006).

[43] B. Surer, M. Troyer, P. Werner, T. O. Wehling, A. M. Läuchli, A. Wilhelm, and A. I. Lichtenstein, Phys. Rev. B 85, 085114 (2012).

[44] P. J. Feibelman, Phys. Rev. B 35, 2626 (1987).

[45] C. Verdozzi, P. A. Schultz, R. Wu, A. H. Edwards, and N. Kioussis, Phys. Rev. B 66, 125408 (2002).

[46] J. P. Perdew, K. Burke, and M. Ernzerhof, Phys. Rev. Lett. 77, 3865 (1996).

[47] J. Perdew, K. Burke, and M. Ernzerhof, Phys. Rev. Lett. 78, 1396 (1997).

[48] S. G. Ovchinnikov and V. V. Val'kov, Hubbard Operators in the Theory of Strongly Correlated Electrons (Imperial College Press, London, UK, 2004).

[49] F. Bonetto, C. Gonzalez, and E. C. Goldberg, Phys. Rev. B 93, 195439 (2016).

[50] S. Huzinaga, J. Chem. Phys. 42, 1293 (1965).

[51] S. Huzinaga, J. Andzelm, M. Klobukowsky, E. RadzioAndzelm, Y. Sakai, and H. Tatewaki, Gaussian Basis Set for Molecular Calculations (Elsevier, Amsterdam, 1984).

[52] J. W. Rabalais, Principles and Applications of Ion Scattering Spectrometry (Wiley-Interscience, New Jersey, 2003).

[53] P. P. Baruselli, R. Requist, A. Smogunov, M. Fabrizio, and E. Tosatti, Phys. Rev. B 92, 045119 (2015).

[54] W. Kohn and L. J. Sham, Phys. Rev. 140, A1133 (1965).

[55] V. M. Silkin, J. Zhao, F. Guinea, E. V. Chulkov, P. M. Echenique, and H. Petek, Phys. Rev. B 80, 121408(R) (2009).

[56] F. Bonetto, M. A. Romero, E. A. García, R. A. Vidal, J. Ferrón, and E. C. Goldberg, Phys. Rev. B 78, 075422 (2008).

[57] A. Radzig and B. Smirnov, Reference Data on Atoms, Molecules, and Ions (Springer, Berlin, Germany, 1985).

[58] K. Xu, C. Zeng, Q. Zhang, R. Yan, P. Ye, K. Wang, A. C. Seabaugh, H. G. Xing, J. S. Suehle, C. A. Richter, D. J. Gundlach, and N. V. Nguyen, Nano Lett. 13, 131 (2013).

[59] S.-J. Liang and L. K. Ang, Phys. Rev. Appl. 3, 014002 (2015).

[60] N. Grewe, T. Jabben, and S. Schmitt, Eur. Phys. J. B 68, 23 (2009).

[61] F. Flores and E. C. Goldberg, J. Phys.: Condens. Matter 29, 055602 (2017).

[62] F. Liu, M. Wang, Y. Chen, and J. Gao, J. Solid State Chem. 276, 100 (2019).

[63] A. E. Galashev and O. R. Rakhmanova, Phys. Usp. 57, 970 (2014).

[64] A.-P. Jauho, N. S. Wingreen, and Y. Meir, Phys. Rev. B 50, 5528 (1994).

[65] J. M. Blanco, C. Gonzalez, P. Jelinek, J. Ortega, F. Flores, and R. Perez, Phys. Rev. B 70, 085405 (2004).

[66] Y. Meir, N. S. Wingreen, and P. A. Lee, Phys. Rev. Lett. 66, 3048 (1991).

[67] Y. Meir and N. S. Wingreen, Phys. Rev. Lett. 68, 2512 (1992).

[68] J. O. Lugo, L. I. Vergara, P. G. Bolcatto, and E. C. Goldberg, Phys. Rev. A 65, 022503 (2002). 